\title{
On p-form gauge theories and their conformal limits
}

\author{
Igor Bandos, ${ }^{a, b}$ Kurt Lechner, ${ }^{c}$ Dmitri Sorokin ${ }^{c}$ and Paul K. Townsend ${ }^{d}$ \\ ${ }^{a}$ Department of Theoretical Physics, University of the Basque Country UPV/EHU, \\ P.O. Box 644, 48080 Bilbao, Spain \\ ${ }^{b}$ IKERBASQUE, Basque Foundation for Science, \\ Plaza Euskadi 5, 48009 Bilbao, Spain \\ cI.N.F.N., Sezione di Padova and Dipartimento di Fisica e Astronomia "Galileo Galilei", \\ Università degli Studi di Padova, \\ Via F. Marzolo 8, 35131 Padova, Italy \\ ${ }^{d}$ Department of Applied Mathematics and Theoretical Physics, \\ Centre for Mathematical Sciences, University of Cambridge, \\ Wilberforce Road, Cambridge, CB3 0WA, U.K. \\ E-mail: igor.bandos@ehu.eus, kurt.lechner@pd.infn.it, \\ dmitri.sorokin@pd.infn.it, P.K.Townsend@damtp.cam.ac.uk
}

ABSTRACT: Relations between the various formulations of nonlinear p-form electrodynamics with conformal-invariant weak-field and strong-field limits are clarified, with a focus on duality invariant $(2 n-1)$-form electrodynamics and chiral $2 n$-form electrodynamics in Minkowski spacetime of dimension $D=4 n$ and $D=4 n+2$, respectively. We exhibit a new family of chiral 2-form electrodynamics in $D=6$ for which these limits exhaust the possibilities for conformal invariance; the weak-field limit is related by dimensional reduction to the recently discovered ModMax generalisation of Maxwell's equations. For $n>1$ we show that the chiral 'strong-field' $2 n$-form electrodynamics is related by dimensional reduction to a new $S l(2 ; \mathbb{R})$-duality invariant theory of $(2 n-1)$-form electrodynamics.

Keywords: Duality in Gauge Field Theories, Brane Dynamics in Gauge Theories, SpaceTime Symmetries

ARXiv EPrint: 2012.09286 


\section{Contents}

1 Introduction $\quad 2$

2 p-form electrodynamics: preliminaries 4

$2.12 \mathrm{D}$ chiral 0-form electrodynamics 5

2.2 4D nonlinear 1-form electrodynamics 6

2.2.1 BB electrodynamics and the Legendre transform 8

$\begin{array}{lll}2.2 .2 & \text { Off-shell duality invariance } & 10\end{array}$

2.3 6D chiral 2-form: Hamiltonian formulation 11

2.3.1 Reduction/truncation to 4D 14

2.4 6D chiral 2-form: Perry-Schwarz formulation $\quad 15$

$\begin{array}{lll}2.4 .1 & 4 \mathrm{D} \text { reduction redux } & 16\end{array}$

$\begin{array}{llr}3 & \text { PST formulation } & 17\end{array}$

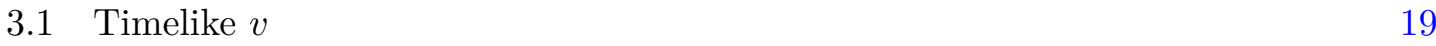

3.1.1 4D reduction/truncation prior to gauge-fixing of $v \quad 20$

$\begin{array}{ll}3.2 & \text { Spacelike } v\end{array} 21$

$\begin{array}{lll}3.3 & \text { Variant PST formulations } & 21\end{array}$

4 New examples in 4D and 6D 22

4.1 ModMax electrodynamics 23

4.2 New chiral 2-form theories 24

$\begin{array}{lll}\text { 4.2.1 PST formulation } & 25\end{array}$

4.2.2 Conformal invariance of the weak/strong-field limits 25

5 Higher dimensions: duality in $D=4 n \quad 27$

$\begin{array}{lll}5.1 & \text { Generalized BI-type electrodynamics } & 29\end{array}$

5.2 Legendre transform redux 30

6 Higher dimensions: chirality in $D=4 n+2$

6.1 Reduction to $D=4 n$

6.1.1 A new $D>4$ generalization of BB electrodynamics 34

$\begin{array}{lll}7 & \text { Summary and discussion } & 35\end{array}$ 


\section{Introduction}

The Born-Infeld (BI) theory of non-linear electrodynamics in four dimensions (4D) and a related non-linear chiral 2-form electrodynamics in six dimensions (6D) arise naturally in String/M-theory as truncations of the effective low-energy dynamics of the D3-brane and M5-brane, respectively. They are related by a consistent dimensional-reduction/truncation inherited from the fact that the D3-brane effective (4D worldvolume) action is a consistent dimensional reduction of the M5-brane effective (6D worldvolume) action [1,2], where 'consistent' means that any solution of the lower-dimensional equations 'lifts' to a solution of the higher-dimensional equations. For this reason, it is useful to consider the 4D BI theory and the 6D chiral 2-form electrodynamics on the M5-brane as partners in what we shall call the "D3/M5 pair".

In this paper we explore the possibilities for other $4 \mathrm{D} / 6 \mathrm{D}$ pairs in the context of various formulations of both the $4 \mathrm{D}$ and $6 \mathrm{D}$ partners. In the $4 \mathrm{D}$ case we consider the generic nonlinear electrodynamics theory that is both Lorentz invariant and invariant under an $\mathrm{SO}(2)$ electromagnetic-duality group (as is the $\mathrm{BI}$ theory). In the $6 \mathrm{D}$ case we consider the general Lorentz invariant nonlinear chiral 2-form electrodynamics; as for the D3/M5 pair, 6D chirality implies electromagnetic-duality of the 4D nonlinear electrodynamics obtained by a consistent reduction/truncation.

Our principal new result is a one-parameter generalization of the D3/M5 pair for which the $4 \mathrm{D}$ partner is the "generalized BI electrodynamics" of [3], which has the property that its weak-field and strong-field limits exhaust the possibilities for conformal duality-invariant $4 \mathrm{D}$ electrodynamics. The $6 \mathrm{D}$ partner is a new interacting familiy of chiral 2 -form electrodynamics theories with the same property: its weak-field and strong-field limits exhaust the possibilities for conformal chiral 2-form electrodynamics. For the $4 \mathrm{D}$ case it was shown in [3] that the strong-field limit is the same as that of the BI theory, i.e. the $\operatorname{Sl}(2 ; \mathbb{R})$-duality invariant Bialynicki-Birula (BB) electrodynamics [4], but the weak-field limit is a novel interacting one-parameter "ModMax" generalization of Maxwell electrodynamics. Here we find that its 6D partner has the same strong-field limit as that of the 'M5' chiral 2-form electrodynamics $[5,6]$ but its weak-field limit is a new conformal chiral 2-form electrodynamics that contains ModMax electrodynamics as a consistent reduction/truncation.

We also explore the possibility of higher-dimensional analogs of these 4D/6D pairs. A class of duality invariant BI-inspired $(2 n-1)$-form electrodynamics theories in a (Minkowski) spacetime of dimension $D=4 n$ was found in [7]. A natural question is whether they are obtainable by consistent truncation/reduction of some chiral $2 n$-form electrodynamics in $D=4 n+2$ for $n>1$. For $n=2$ (at least) the answer is no, because the leading (quartic) interaction terms in the weak-field expansion do not match those found by reduction/truncation of the quartic interaction terms in the weak-field expansion of any 10D chiral 4-form electrodynamics that has a weak-field expansion [8,9]. This result leaves open the possibility that the higher-dimensional generalization of BB electrodynamics found in [10] from the strong-field limit of the BI-type theory of Gibbons and Rasheed [7] is a consistent truncation of some analogous higher-dimensional generalization of the strong-field 'M5' chiral 2-form electrodynamics. 
In fact, there is a natural generalization of the strong-field 'M5' electrodynamics to an interacting conformal chiral $2 n$-form electrodynamics in $D=4 n+2$ for any $n>1$ [11] and we find here, by reduction/truncation, the corresponding conformal duality-invariant $(2 n-1)$-form electrodynamics in $D=4 n$; for $n=1$ this is BB electrodynamics but for $n>1$ it is a new higher-dimensional generalization that differs from that of [10]. This is possible because for $n>1$ the requirements of conformal invariance and $S l(2 ; \mathbb{R})$-duality invariance do not determine the Hamiltonian density uniquely.

Another purpose of this paper is to explore the relations between the various formulations of the generic p-form electrodynamics theories mentioned above, mostly focusing on the $4 \mathrm{D}$ and $6 \mathrm{D}$ cases. In the $4 \mathrm{D}$ case, we have the standard Lagrangian and Hamiltonian formulations of generic nonlinear duality-invariant (1-form) electrodynamics but in neither formulation are both Lorentz invariance and duality invariance manifest. In the $6 \mathrm{D}$ case we have a Hamiltonian formulation $[5,6,10,12,13]$, which we develop further here. However, because of chirality [14], the closest one can get to a standard Lagrangian formulation is what we call here the Perry-Schwarz formulation in which only a 5D Lorentz invariance is manifest [15]; this is essentially a variant of the Hamiltonian formulation for which the manifest Lorentz symmetry subgroup is $\mathrm{SO}(1,4)$ rather than $\mathrm{SO}(5)$. For both $4 \mathrm{D}$ and $6 \mathrm{D}$ there $i s$ a formulation in which all symmetries are manifest; this is the PST formulation, which involves an additional closed 1-form field [16-18], but an additional non-manifest gauge invariance is then needed for equivalence with the 'standard' formulations.

A consequence of the fact that not all symmetries (and gauge-invariances) can be simultaneously manifest is that there is always some symmetry (or gauge invariance) that must be imposed. This means that the function defining the particular model (e.g. Hamiltonian density in the Hamiltonian formulation) must satisfy a condition, and in each case this can be expressed as a partial differential equation (PDE) in two independent variables (e.g. two independent rotation scalars for the Hamiltonian density). In the 4D case this PDE has been found various times using various methods $[4,7,19,20]$, and the same is true for $6 \mathrm{D}[15,21,22]$. For an appropriate choice of the two independent variables in each case, the PDE is the same in all cases: not only do different formulations of the $4 \mathrm{D}$ or $6 \mathrm{D}$ theories lead to the same PDE, but also (as observed in [15]) one finds the same PDE for both $4 \mathrm{D}$ and $6 \mathrm{D}$. Here we explain this result by showing how the 6D PDE is mapped into the $4 \mathrm{D}$ PDE by the process of consistent reduction/truncation. This implies a one-to-one correspondence between $4 \mathrm{D}$ nonlinear theories of duality-invariant electrodynamics and 6D nonlinear chiral 2-form electrodynamics.

We shall begin with a review of the $p=0,1,2$ cases of $p$-form electrodynamics in a Minkowski spacetime of dimension $D=2 p+2$; these are the dimensions that allow either electromagnetic duality invariance (for odd $p$ ) or chirality (for even $p$ ). This review includes some new material; for example we recover by Hamiltonian methods the result of [9] that any chiral 0-form electrodynamics is a free-field theory, and we give another derivation of the condition on the Hamiltonian density for Lorentz invariance of the generic $6 \mathrm{D}$ chiral 2-form electrodynamics. We also explain how unusual features of the Legendre transform for BB-electrodynamics do not prevent an equivalence of the Hamiltonian and Lagrangian formulations even though the (canonical) Lagrangian density is identically zero. These 
"preliminaries" are followed by an exposition of the PST method in which some details passed over in earlier works on this topic are explained.

The abstract 'universal' PDE that one must solve to find any particular pair of duality invariant $4 \mathrm{D}$ electrodynamics and chiral 2 -form $6 \mathrm{D}$ electrodynamics has a known general solution [23] but its application to electrodynamics (see e.g. [7, 15, 24]) requires additional physical constraints, such as the requirement of an 'acceptable' weak-field limit. It has generally been supposed that the weak-field limit must be a free-field theory, but it was argued in [3] that the ModMax theory mentioned above is a physically acceptable alternative weakfield limit for $4 \mathrm{D}$ electrodynamics; here we give some details of the derivation of the oneparameter generalization of BI theory that has ModMax as its weak-field limit. The principal novelty is its $6 \mathrm{D}$ analog (and its weak-field limit) which is the new family of chiral 2-form electrodynamics advertised in our Abstract; we also provide an alternative proof of conformal invariance of the weak-field and strong-field limits that applies both in 4D and $6 \mathrm{D}$.

We then turn to the higher-dimension $p$-form theories, using their PST formulation to obtain the results mentioned above. In addition, we discuss the Legendre transform for a class of (odd- $p$ ) duality-invariant $p$-form electrodynamics theories that include the "generalized BI" theory of [3]. There we showed how the weak-field limits are related by a Legendre transform; here we use a more powerful method that not only avoids the need to take the weak-field limit but also applies for any odd $p \geq 1$.

We conclude with an overview of the paper and some discussion of open problems.

\section{2 p-form electrodynamics: preliminaries}

By "p-form electrodynamics" we mean here an abelian gauge-field theory for a $p$-form potential $A$ with $(p+1)$-form field-strength $F=d A$, in a Minkowski spacetime of dimension $2 p+2$. For $p$ odd, $F$ transforms irreducibly with respect to the Lorentz group. In contrast, $F=F_{+} \oplus F_{-}$for even $p$, where $F_{ \pm}$are the (anti)self-dual components of $F$, which transform as distinct irreducible representations ${ }^{1}$ that are exchanged by parity; in this case we may set $F=F_{+}$to get a "chiral" theory. For any $p$ there could be $(p-1)$-brane sources, which may be 'electric' or magnetic' (or 'dyonic') for odd $p$, but we consider here only source-free theories.

We also assume that the Lagrangian density is an ultralocal Lorentz-scalar function of $F$; i.e. it does not involve any derivatives of $F$. This means that the dimension of the space of initial conditions at any space point is unaffected by interactions; in other words, interactions allowed by this assumption do not change the number of degrees of freedom. In the context of the Hamiltonian formulation, this implies that the canonical structure is unaffected by the interactions.

The lowest odd $p$ is $p=1$; the free field case is Maxwell electrodynamics and the bestknown interacting example is Born-Infeld electrodynamics. The lowest even $p$ is $p=0$; the free field case is the "chiral boson". Self-interactions are not possible for $p=0[9]$; here we give another proof of this statement. The next-to-lowest even $p$ is $p=2$, and this

\footnotetext{
${ }^{1}$ We may also write $F=F_{+}+F_{-}$for odd p but then $F_{-}=F_{+}^{*}$ for real $F$, so we do not have a decomposition of $F$ into irreducible representations of the Lorenz group.
} 
includes not only the free chiral 2-form electrodynamics but also the BI-type theory on the M5-brane.

As mentioned in the introduction, the $p=1$ and $p=2$ cases are linked by dimensional reduction, so it is convenient to consider them together. The main aim of this section is to present the basic properties of these theories from both a Lagrangian and a Hamiltonian perspective; much of this will be review but some known results are recovered by different methods. Our aim is to exhibit the unity underlying the different formulations of p-form electrodynamics subject to a duality-invariance/chirality restriction.

\section{$2.12 \mathrm{D}$ chiral 0-form electrodynamics}

In this case $F=d \varphi$ for a scalar field $\varphi(\tau, \sigma)$. The chirality condition on $F$ is $\dot{\varphi}=\varphi^{\prime}$ (with $\dot{\varphi} \equiv \partial_{\tau} \varphi$ and $\left.\varphi^{\prime} \equiv \partial_{\sigma} \varphi\right)$, which is the standard chiral boson equation. However, to address the issue of possible interactions in a way that is in line with the general definition above of p-form electrodynamics it is convenient to start from a Hamiltonian formulation in which the Hamiltonian field equations follow from variation of the phase-space (or canonical) action

$$
I=\int d t \int d \sigma\{\mp \dot{\varphi} \psi-\mathcal{H}(\psi)\}, \quad \psi=\varphi^{\prime},
$$

where an overdot indicates a time derivative and a prime indicates a space derivative. We allow the Hamiltonian density to be an arbitrary function of the 'magnetic' field $\psi$, which is invariant under the 'semi-local' gauge transformation $\varphi \rightarrow \varphi+\alpha(t)$, where $\alpha$ is an arbitrary function of time. The field equation is

$$
\dot{\varphi}^{\prime}=\mp \frac{1}{2}\left(\mathcal{H}_{\psi}\right)^{\prime}
$$

The action (2.1) is manifestly invariant under translations in time and space, and the corresponding Noether charges are

$$
H=\int d \sigma \mathcal{H}, \quad P= \pm \int d \sigma \psi^{2} .
$$

These are time-independent (for suitable boundary conditions) since the field equation implies that

$$
\partial_{t} \mathcal{H}=\mp \frac{1}{4}\left(\mathcal{H}_{\psi}^{2}\right)^{\prime}, \quad \partial_{t}\left[\psi^{2}\right]=\mp\left(\mathcal{H}-\psi \mathcal{H}_{\psi}\right)^{\prime} .
$$

If the action (2.1) is Lorentz invariant then the Lorentz boost Noether charge is

$$
L=t P-\int d \sigma \sigma \mathcal{H}
$$

However, we must check that this is a conserved charge because the action is not manifestly Lorentz invariant. Using the field equation (2.2), and assuming that boundary terms are zero, we have

$$
\dot{L}=P-\int d \sigma \sigma \dot{\mathcal{H}}= \pm \int d \sigma\left(\psi-\frac{1}{2} \mathcal{H}_{\psi}\right)\left(\psi+\frac{1}{2} \mathcal{H}_{\psi}\right)
$$


which is zero iff $\mathcal{H}_{\psi}= \pm 2 \psi$. We may assume without loss of generality that $\mathcal{H}$ is nonnegative, and zero for $\psi=0$, in which case

$$
\mathcal{H}(\psi)=\psi^{2}
$$

We thus conclude that the most general Lorentz invariant action of the form (2.1) is

$$
I=\int d t \int d \sigma\left(\mp \dot{\varphi}-\varphi^{\prime}\right) \varphi^{\prime} .
$$

This is the Floreanini-Jackiw action for a free (anti)chiral boson [25]. In agreement with [9] we conclude that no Lorentz invariant self-interactions are possible.

\section{$2.24 \mathrm{D}$ nonlinear 1 -form electrodynamics}

We now review aspects of nonlinear theories of electrodynamics in a 4D Minkowski spacetime. A convenient starting point is the phase-space action

$$
I=\int d t \int d^{3} \sigma\{\mathbf{E} \cdot \mathbf{D}-\mathcal{H}(\mathbf{D}, \mathbf{B})\},
$$

where $(t, \boldsymbol{\sigma})$ are the time and cartesian space coordinates. The electric field $\mathbf{E}$ and magnetic induction field $\mathbf{B}$ are defined as

$$
\mathbf{E}=\nabla A_{0}-\dot{\mathbf{A}}, \quad \mathbf{B}=\boldsymbol{\nabla} \times \mathbf{A},
$$

which means (in this context) that the electric-displacement 3 -vector $\mathbf{D}$ is canonically conjugate to minus the vector potential $\mathbf{A}$, while the scalar potential $A_{0}$ is a Lagrange multiplier for the constraint $\boldsymbol{\nabla} \cdot \mathbf{D}=0$. If we choose the Hamiltonian density to be a function of the three independent rotation scalars

$$
s=\frac{1}{2}\left(|\mathbf{D}|^{2}+|\mathbf{B}|^{2}\right), \quad \xi=\frac{1}{2}\left(|\mathbf{D}|^{2}-|\mathbf{B}|^{2}\right), \quad \eta=\mathbf{D} \cdot \mathbf{B},
$$

then the action is invariant under time and space translations, and rotations. It is also Lorentz invariant if [4]

$$
\mathcal{H}_{s}^{2}-\mathcal{H}_{\xi}^{2}-\mathcal{H}_{\eta}^{2}=1
$$

The Hamiltonian density is further invariant under an $\mathrm{SO}(2)$ 'duality' rotation of the 2 -vector with 3-vector components $(\mathbf{D}, \mathbf{B})$ if it is a function only of $s$ and

$$
p=|\mathbf{D} \times \mathbf{B}|,
$$

which is related to $(s, \xi, \eta)$ by

$$
s^{2}-p^{2}=\xi^{2}+\eta^{2} .
$$

In terms of these variables the condition for Lorentz invariance (2.12) becomes

$$
\mathcal{H}_{s}^{2}+\frac{2 s}{p} \mathcal{H}_{s} \mathcal{H}_{p}+\mathcal{H}_{p}^{2}=1
$$


As $p$ has an $S l(2 ; \mathbb{R})$ electromagnetic duality invariance, the same will be true of $\mathcal{H}$ iff it is a function only of $p$, and then Lorentz invariance requires $\mathcal{H}= \pm p$. Choosing the positive sign, we have the interacting conformal electrodynamics of Bialynicki-Birula [4]

$$
\mathcal{H}_{B B}=|\mathbf{D} \times \mathbf{B}| .
$$

An alternative basis for $\mathrm{SO}(2)$ duality invariant rotation scalars is

$$
\mathrm{u}=\frac{1}{2}\left(s+\sqrt{s^{2}-p^{2}}\right), \quad \mathrm{v}=\frac{1}{2}\left(s-\sqrt{s^{2}-p^{2}}\right) .
$$

These new variables are well defined since it follows from (2.14) that $s^{2}-p^{2} \geq 0$. In terms of them, the Lorentz-invariance condition (2.15) simplifies to

$$
\mathcal{H}_{\mathrm{u}} \mathcal{H}_{\mathrm{v}}=1
$$

Remarkably, an equation formally equivalent to (2.18) appears in the manifestly Lorentz-invariant Lagrangian formulation as the condition for electromagnetic duality invariance $[7,19,24,26-32]$. The Lagrangian density $\mathcal{L}$ of a general Lorentz invariant theory of electrodynamics may be written in terms of the two independent Lorentz scalars

$$
S=-\frac{1}{4} F_{\mu \nu} F^{\mu \nu}=\frac{1}{2}\left(|\mathbf{E}|^{2}-|\mathbf{B}|^{2}\right), \quad P=-\frac{1}{8} \varepsilon^{\mu \nu \lambda \rho} F_{\mu \nu} F_{\lambda \rho}=\mathbf{E} \cdot \mathbf{B} .
$$

In the Lagrangian formulation, the electric-displacement vector $\mathbf{D}$ is defined as $\partial \mathcal{L} / \partial \mathbf{E}$, and the condition for electromagnetic-duality invariance of the EL equations of a Lagrangian density $\mathcal{L}(S, P)$ is

$$
\mathcal{L}_{S}^{2}-\frac{2 S}{P} \mathcal{L}_{S} \mathcal{L}_{P}-\mathcal{L}_{P}^{2}=1
$$

which is very similar to $(2.15)$. In terms of the new variables

$$
U=\frac{1}{2}\left(S-\sqrt{S^{2}+P^{2}}\right), \quad V=\frac{1}{2}\left(S+\sqrt{S^{2}+P^{2}}\right)
$$

this duality-invariance condition simplifies to [7]

$$
\mathcal{L}_{U} \mathcal{L}_{V}=1,
$$

which is formally identical to (2.18).

To summarise, the Hamiltonian and Lagrangian densities of a generic non-linear Lorentz and duality invariant electrodynamics theory are both solutions of the same PDE for particular choices of the two pairs of variables on which they depend. For any $\mathcal{H}(\mathrm{u}, \mathrm{v})$ satisfying (2.18) its Legendre transform (with respect to $\mathbf{D}$ ) will be some $\mathcal{L}(U, V)$ satisfying (2.22) [20]. For example, one solution to the equation (2.18) is

$$
\begin{aligned}
\mathcal{H}_{(T)} & =\sqrt{T^{2}+2 T(\mathrm{u}+\mathrm{v})+4 \mathrm{uv}}-T \\
& =\sqrt{T^{2}+T\left(|\mathbf{D}|^{2}+|\mathbf{B}|^{2}\right)+|\mathbf{D} \times \mathbf{B}|^{2}}-T,
\end{aligned}
$$

which is the BI Hamiltonian density. The corresponding solution to (2.22) is

$$
\begin{aligned}
\mathcal{L}_{(T)} & =T-\sqrt{T^{2}-2 T(U+V)+4 U V} \\
& =T-\sqrt{T^{2}-T\left(|\mathbf{E}|^{2}-|\mathbf{B}|^{2}\right)-(\mathbf{E} \cdot \mathbf{B})^{2}},
\end{aligned}
$$

which is the BI Lagrangian density. 


\subsubsection{BB electrodynamics and the Legendre transform}

Starting with the BI Hamiltonian density of (2.23) we may, following Bialynicki-Birula [4], take the $T \rightarrow 0$ limit to arrive at the BB Hamiltonian density of (2.16):

$$
\lim _{T \rightarrow 0} \mathcal{H}_{(T)}(\mathrm{u}, \mathrm{v})=2 \sqrt{\mathrm{uv}}=|\mathbf{D} \times \mathbf{B}|=\mathcal{H}_{B B} .
$$

As $T$ has dimensions of energy density, this limit should be seen as a strong-field limit in which the energy density is much greater than $T$, just as the weak-field limit should be seen as the limit in which the energy density is much less than $T$. One might expect these limits to yield conformal invariant theories, as is evidently true for the weak field limit since the Hamiltonian equations in this limit are Maxwell's equations. As shown in [4], it is also true for the strong-field limit, which has the additional feature that $\mathcal{H}_{B B}$ is invariant under an $S l(2 ; \mathbb{R})$ electromagnetic-duality acting on the 3 -vector-valued 2 -vector $(\mathbf{D}, \mathbf{B})$.

Let us find the Lagrangian density of this BB-electrodynamics by taking the Legendre transform of $\mathcal{H}_{B B}$; the first step is to define

$$
\mathbf{E}:=\frac{\partial \mathcal{H}_{\mathcal{B B}}}{\partial \mathbf{D}}=-\mathbf{n} \times \mathbf{B}, \quad \mathbf{n}=\frac{\mathbf{D} \times \mathbf{B}}{|\mathbf{D} \times \mathbf{B}|} .
$$

The 'canonical' BB Lagrangian density is then defined as

$$
\mathcal{L}_{B B}(\mathbf{E}, \mathbf{B}):=\sup _{\mathbf{D}}\left[\mathbf{D} \cdot \mathbf{E}-\mathcal{H}_{B B}\right]
$$

In principle, this requires us to find the $\mathbf{D}$ that maximises the expression in brackets. However, in this case

$$
\mathbf{D} \cdot \mathbf{E}-\mathcal{H}_{B B}=\mathbf{n} \cdot(\mathbf{D} \times \mathbf{B})-|\mathbf{D} \times \mathbf{B}| \equiv 0,
$$

so we conclude, following [4], that

$$
\mathcal{L}_{B B}(\mathbf{E}, \mathbf{B}) \equiv 0
$$

Despite this conclusion it remains true that the Legendre transform of $\mathcal{L}_{B B}$ is $\mathcal{H}_{B B}$. We shall provide an explicit proof below, but we first wish to point out that it is a consequence of the convexity of $\mathcal{H}_{B B}$ as a function of $\mathbf{D}$ since a general theorem (see e.g. [33]) guarantees that the Legendre transform defined as in (2.27) (but now with $\mathcal{L}_{B B}$ and $\mathcal{H}_{B B}$ exchanged) is an involution when acting on convex functions. So all we really need to prove is convexity of $\mathcal{H}_{B B}$ and we can do this by investigating its Hessian matrix:

$$
\frac{\partial^{2} \mathcal{H}_{B B}}{\partial D^{i} \partial D^{j}}=\frac{|\mathbf{B}|^{2}}{\mathcal{H}_{B B}} n_{i} n_{j}
$$

As all eigenvalues of this matrix are non-negative for all $\mathbf{D}$, given any $\mathbf{B}$, the function $\mathcal{H}_{B B}$ is convex. This is sufficient for the theorem, but the case under consideration is special because $\mathcal{H}_{B B}$ is not strictly convex (some eigenvalues of its Hessian are zero) and a consequence of this is that we cannot solve unambiguously for $\mathbf{D}$ the equation defining $\mathbf{E}$, 
i.e. (2.26). A corollary is that the equation defining $\mathbf{E}$ imposes constraints on $\mathbf{E}$; from (2.26) we see that these constraints are ${ }^{2}$

$$
S=P=0,
$$

where $(S, P)$ are the Lorentz scalars of (2.19). In other words, although $\mathcal{L}_{B B}$ is identically zero the domain of the $\mathbf{E}$ field-space in which it is defined is restricted by $S=P=0$. This means that the Legendre transform of $\mathcal{L}_{B B}$ is

$$
\mathcal{H}(\mathbf{D}, \mathbf{B})=\sup _{\{\mathbf{E} \mid S=P=0\}}[\mathbf{D} \cdot \mathbf{E}-0] .
$$

Now we have a constrained variational problem: we must find the $\mathbf{E}$, within its allowed domain, that maximises the expression in brackets.

We can solve this constrained variational problem by the Lagrange multiplier method; i.e. we first look for the stationary points of

$$
\mathcal{H}(\mathbf{D}, \mathbf{B} ; \mathbf{E}, \lambda, \mu):=\mathbf{D} \cdot \mathbf{E}-\lambda S-\mu P,
$$

where we must vary $\mathbf{E}$ (now without constraints) and the Lagrange multiplier fields $(\lambda, \mu)$. We must then examine the results of this calculation to find the maximum of $\mathbf{D} \cdot \mathbf{E}$, rather than a minimum or some other stationary value.

Varying the function defined in (2.33) with respect to $\mathbf{E}$ we have $\mathbf{E}=\lambda^{-1}(\mathbf{D}-\mu \mathbf{B})$, and back-substitution yields

$$
\mathcal{H}(\mathbf{D}, \mathbf{B} ; \lambda, \mu)=\frac{1}{2 \lambda}|\mathbf{D}-\mu \mathbf{B}|^{2}+\frac{\lambda}{2}|\mathbf{B}|^{2} .
$$

Varying this with respect to $\mu$ we have $\mu|\mathbf{B}|^{2}=\mathbf{D} \cdot \mathbf{B}$ and back-substitution yields

$$
\mathcal{H}(\mathbf{D}, \mathbf{B} ; e)=\frac{1}{2}\left[e^{-1}|\mathbf{D} \times \mathbf{B}|^{2}+e\right], \quad e=\lambda|\mathbf{B}|^{2} .
$$

Finally, elimination of $e$ yields $e= \pm|\mathbf{D} \times \mathbf{B}|$ and hence

$$
\mathcal{H}(\mathbf{D}, \mathbf{B})= \pm|\mathbf{D} \times \mathbf{B}|
$$

for unrestricted $\mathbf{D}$. The maximum is achieved by choosing the top sign, so the Legendre transform of $\mathcal{L}_{B B}$ is $\mathcal{H}_{B B}$, as claimed.

The unusual aspect of this particular pairing of convex functions by the Legendre transform is that the information in $\mathcal{L}_{B B}$ that is needed for the reconstruction of $\mathcal{H}_{B B}$ resides entirely in the restriction on its domain, not on its values within this domain!

Finally, we should point out that the effect of using the Lagrange multiplier method to solve the constrained variation problem, implicit in the definition (2.32) of $\mathcal{H}_{B B}$ as the Legendre transform of $\mathcal{L}_{B B}$, is to replace the identically zero canonical Lagrangian density by its "weak" equivalent ${ }^{3}$

$$
\mathcal{L}_{B B} \approx \lambda S+\mu P
$$

This is precisely the Lagrangian density proposed in [35], where it was verified that the field equations are equivalent to the Hamiltonian field equations found from $\mathcal{H}_{B B}$. Now we can see more precisely why this is true.

\footnotetext{
${ }^{2}$ The $T \rightarrow 0$ limit of the BI Lagrangian density may be taken if $P=0$ is imposed, with the result that $\mathcal{L}_{B B}=0$, but this attempt to take the strong-field limit misses the $S=0$ constraint.

${ }^{3}$ In the terminology of constrained Hamiltonian systems [34].
} 


\subsubsection{Off-shell duality invariance}

For the phase-space action (2.9), any invariance of the Hamiltonian density will be an invariance of the Hamiltonian field equations but not necessarily of the action itself; if the action is not invariant we have an "on-shell" symmetry. Electromagnetic duality invariance, acting as a $\mathrm{SO}(2)$ transformation on $(\mathbf{D}, \mathbf{B})$, is an example; it is an "on-shell" symmetry when $\mathcal{H}$ is duality invariant but it cannot be an "off-shell" symmetry of the action (2.9) because $\mathbf{B}=\boldsymbol{\nabla} \times \mathbf{A}$ is identically divergence-free but its duality partner $\mathbf{D}$ is divergencefree only as the result of a constraint imposed by a Lagrange multiplier. However, this "off-shell" difference between $\mathbf{D}$ and $\mathbf{B}$ may be eliminated, in the absence of sources, by solving the constraint on $\mathbf{D}$ in terms of a new 'dual' vector potential $\tilde{\mathbf{A}}$ :

$$
\mathbf{D}=\nabla \times \tilde{\mathbf{A}} .
$$

This replacement converts the action (2.9) into one that is a functional of the pair of vector potentials $(\mathbf{A}, \tilde{\mathbf{A}})$ :

$$
I[\mathbf{A}, \tilde{\mathbf{A}}]=\int d t \int d^{3} \sigma\{-\dot{\mathbf{A}} \cdot \nabla \times \tilde{\mathbf{A}}-\mathcal{H}(\mathbf{D}, \mathbf{B})\} .
$$

We now have both $\boldsymbol{\nabla} \cdot \mathbf{D}=0$ and $\boldsymbol{\nabla} \cdot \mathbf{B}=0$ as identities, while variation with respect to $\mathbf{A}$ and $\tilde{\mathbf{A}}$ yields the remainder of the "macroscopic Maxwell equations":

$$
\dot{\mathbf{B}}=-\nabla \times \mathbf{E}, \quad \dot{\mathbf{D}}=\nabla \times \mathbf{H},
$$

where $(\mathbf{E}, \mathbf{H})$ are again given by the constitutive relations:

$$
\mathbf{E}=\frac{\partial \mathcal{H}}{\partial \mathbf{D}}, \quad \mathbf{H}=\frac{\partial \mathcal{H}}{\partial \mathbf{B}} .
$$

Lorentz invariance is not guaranteed; the condition for it is [4]

$$
\mathbf{E} \times \mathbf{H}=\mathbf{D} \times \mathbf{B} .
$$

Electromagnetic duality invariance is not guaranteed either but it now acts as an $\mathrm{SO}(2)$ rotation of the vector-potential doublet $(\mathbf{A}, \tilde{\mathbf{A}})$, and is a symmetry of the action (2.39) if $\mathcal{H}$ is an $\mathrm{SO}(2)$ invariant; equivalently, if [4]

$$
\mathbf{E} \cdot \mathbf{B}=\mathbf{H} \cdot \mathbf{D} .
$$

The relations (2.42) and (2.43) are jointly equivalent to the PDE (2.15) to be satisfied by $\mathcal{H}$ and, as expected, (2.43) is an identity when $\mathcal{H}=\mathcal{H}(s, p)$. An advantage of this formulation is that duality is now an off-shell symmetry. Noether's theorem therefore applies and there is a corresponding conserved Noether charge [4,36]. This method was used in [37] to construct an $S l(2 ; \mathbb{R})$-invariant action for Bialynicki-Birula electrodynamics and thereby find expressions for its $S l(2 ; \mathbb{R})$ triplet of conserved Noether charges.

If we further change notation by setting $(\tilde{\mathbf{A}}, \mathbf{A})=\left(\mathbf{A}^{1}, \mathbf{A}^{2}\right)$, then

$$
(\mathbf{D}, \mathbf{B})=\left(\mathbf{B}^{1}, \mathbf{B}^{2}\right), \quad(-\mathbf{H}, \mathbf{E})=\left(\mathbf{E}^{1}, \mathbf{E}^{2}\right)
$$


where, for $\mathrm{a}=1,2$,

$$
\mathbf{E}^{\mathrm{a}}=\nabla A_{0}^{\mathrm{a}}-\dot{\mathbf{A}}^{\mathrm{a}}, \quad \mathbf{B}^{\mathrm{a}}=\boldsymbol{\nabla} \times \mathbf{A}^{\mathrm{a}} .
$$

Notice that these fields are invariant under the following gauge transformations with an $\mathrm{SO}(2)$-doublet of scalar parameters $\alpha^{\mathrm{a}}$ :

$$
A_{0}^{\mathrm{a}} \rightarrow A_{0}^{\mathrm{a}}+\dot{\alpha}^{\mathrm{a}}, \quad \mathbf{A}^{\mathrm{a}} \rightarrow \mathbf{A}^{\mathrm{a}}+\nabla \alpha^{\mathrm{a}} .
$$

Notice too that the definitions (2.45) imply the identities

$$
\dot{\mathbf{B}}^{\mathrm{a}} \equiv-\nabla \times \mathbf{E}^{\mathrm{a}}, \quad \nabla \cdot \mathbf{B}^{\mathrm{a}} \equiv 0,
$$

which are the macroscopic Maxwell equations. We must look to the action to find the constitutive relations.

Ignoring surface terms, we may rewrite the action $(2.39)$ as $[20,38]$

$$
I\left[\mathbf{A}^{1}, \mathbf{A}^{2}\right]=\int d t \int d^{3} \sigma\left\{-\frac{1}{2} \epsilon_{\mathrm{ab}} \mathbf{E}^{\mathrm{a}} \cdot \mathbf{B}^{\mathrm{b}}-\mathcal{H}\left(\mathbf{B}^{1}, \mathbf{B}^{2}\right)\right\} .
$$

In addition to being invariant under the gauge transformations (2.46), this action is also invariant under the following gauge transformation with another $\mathrm{SO}(2)$-doublet of scalar parameters $\phi^{\mathrm{a}}$ :

$$
A_{0}^{\mathrm{a}} \rightarrow A_{0}^{\mathrm{a}}+\phi^{\mathrm{a}}
$$

This is because the scalar potentials contribute only to a surface term in the action, which means that the field equations are found from variation of the vector potentials. Because of the identities (2.47), these field equations are equivalent to

$$
\boldsymbol{\nabla} \times\left(\mathbf{E}^{\mathrm{a}}+\epsilon^{\mathrm{ab}} \frac{\partial \mathcal{H}}{\partial \mathbf{B}^{\mathrm{b}}}\right)=\mathbf{0} .
$$

The gauge invariance (2.49) means that the electric fields $\mathbf{E}^{a}$ are, in this context, only defined up to the addition of the gradients $\boldsymbol{\nabla} \phi^{a}$, so the general solution of the field equations (2.50) is gauge-equivalent to

$$
\mathbf{E}^{a}=-\epsilon^{a b} \frac{\partial \mathcal{H}}{\partial \mathbf{B}^{b}}
$$

These are the constitutive relations.

Finally, we observe that in this new notation, the conditions (2.42) and (2.43) for Lorentz and duality invariance, respectively, are now

$$
\epsilon_{\mathrm{ab}}\left(\mathbf{B}^{\mathrm{a}} \times \mathbf{B}^{\mathrm{b}}-\frac{\partial \mathcal{H}}{\partial \mathbf{B}^{\mathrm{a}}} \times \frac{\partial \mathcal{H}}{\partial \mathbf{B}^{\mathrm{b}}}\right)=\mathbf{0}, \quad \epsilon_{\mathrm{ab}}\left(\mathbf{B}^{\mathrm{a}} \times \frac{\partial \mathcal{H}}{\partial \mathbf{B}^{\mathrm{b}}}\right)=0 .
$$

\subsection{D chiral 2-form: Hamiltonian formulation}

We now replace the 3 -vector potential $\mathbf{A}$ by an antisymmetric tensor potential $\mathbb{A}$, with components $\left\{A_{i j} ; i, j,=1, \ldots, 5\right\}$. The analogs of the electric field $\mathbf{E}$ and magnetic-induction field $\mathbf{B}$ are antisymmetric tensors $\mathbb{E}, \mathbb{B}$, with components

$$
E_{i j}=2 \partial_{[i} A_{j] 0}+\dot{A}_{i j}, \quad B^{i j}=\frac{1}{2} \varepsilon^{i j k l m} \partial_{k} A_{l m}:=(\nabla \times \mathbb{A})^{i j} .
$$


For a chiral theory, $\mathbb{B}$ is also the variable canonically conjugate to $\mathbb{A}$, and the constraint imposed by $A_{j 0}$ is an identity, so the action analogous to $(2.9)$ is

$$
I=\int d t \int d^{5} \sigma\left\{\frac{1}{4} \dot{A}_{i j} B^{i j}-\mathcal{H}(\mathbb{B})\right\} .
$$

The normalization of the first term differs from that used in [6] but is more convenient for current purposes. It implies the Poisson-bracket relation

$$
\left\{B^{i j}(\boldsymbol{\sigma}), B^{k l}(\tilde{\boldsymbol{\sigma}})\right\}_{P B}=\varepsilon^{i j k l p} \partial_{p} \delta(\boldsymbol{\sigma}-\tilde{\boldsymbol{\sigma}}) .
$$

The field equation obtained by variation of $\mathbb{A}$ is

$$
\dot{\mathbb{B}}=\nabla \times \mathbb{H}, \quad \mathbb{H} \equiv \frac{\partial \mathcal{H}}{\partial \mathbb{B}} .
$$

The antisymmetric-tensor field $\mathbb{H}$ is the $6 \mathrm{D}$ analog of the magnetic field $\mathbf{H}$ of non-linear $4 \mathrm{D}$ electrodynamics.

The field equation (2.56) implies that

$$
\begin{aligned}
\partial_{t} \mathcal{H} & =\boldsymbol{\nabla} \cdot(\mathbb{H} \times \mathbb{H}), \\
\partial_{t}(\mathbb{B} \times \mathbb{B})_{i} & =\partial_{k}\left[2 H_{i j} B^{j k}-\delta_{i}^{k}(\operatorname{tr}(\mathbb{B H})+2 \mathcal{H})\right],
\end{aligned}
$$

where $^{4}$

$$
(\mathbb{B} \times \mathbb{B})_{i}=\frac{1}{8} \varepsilon_{i j k l m} B^{j k} B^{l m} .
$$

These equations imply that the following integrals are constants of the motion (if surface terms are assumed to vanish):

$$
H=\int d^{5} \sigma \mathcal{H}, \quad \boldsymbol{P}=-\int d^{5} \sigma(\mathbb{B} \times \mathbb{B}) .
$$

These are the Noether charges associated, respectively, to the time translation and space translation invariances of the action. As a check on this interpretation, and the normalizations, one may verify that for any smooth function $f$ of $\mathbb{B}$ satisfying the field equation (2.56), and constants $\left(\alpha^{0}, \alpha^{i}\right)$,

$$
\left\{f, \int d^{5} \sigma\left[\alpha^{0} \mathcal{H}+\alpha^{i}(\mathbb{B} \times \mathbb{B})_{i}\right]\right\}_{P B}=\left[\alpha^{0} \partial_{t}+\alpha^{i} \partial_{i}\right] f .
$$

This shows that the integrals of $\mathcal{H}$ and $\mathbb{B} \times \mathbb{B}$ are the components of a 6 -covector. If we normalize this co-vector such that the dual 6 -vector has the Noether charge $H$ as its time component then its 5 -space component is the Noether charge $\boldsymbol{P}$, irrespective of the signature convention chosen for the Minkowski metric relating 6-vectors to 6-covectors. It then follows that the Noether charge associated to rotational invariance is

$$
J^{i j}=-2 \int d^{5} \sigma \sigma^{[i}(\mathbb{B} \times \mathbb{B})^{j]},
$$

\footnotetext{
${ }^{4}$ The different normalization from [6] compensates for the different normalization of the symplectic form defined by the phase-space action.
} 
which is time-independent, for appropriate boundary conditions, as a consequence of the second of equations (2.57).

For the subset of the chiral 2-form theories defined by $\mathcal{H}(s, p)$, those that are Lorentz invariant will have, as an additional Noether charge, the Lorentz boost generator

$$
\boldsymbol{L}=t \boldsymbol{P}-\int d^{5} \sigma \boldsymbol{\sigma} \mathcal{H} .
$$

A calculation, using the first of eqs. (2.57), yields

$$
\dot{\boldsymbol{L}}=\boldsymbol{P}+\int d^{5} \sigma(\mathbb{H} \times \mathbb{H}) .
$$

From the expression for $\boldsymbol{P}$ in (2.59) we see that $\dot{\boldsymbol{L}}=\mathbf{0}$ requires

$$
\mathbb{H} \times \mathbb{H}=\mathbb{B} \times \mathbb{B} .
$$

As a check on the interpretation of the 5 -vector Noether charge $\boldsymbol{L}$, we may compute the Poisson brackets of its components. Using (2.60), and ignoring surface terms, we find that

$$
\begin{aligned}
\left\{L^{i}, L^{j}\right\}_{P B} & =\iint d^{5} \sigma d^{5} \tilde{\sigma} \sigma^{i} \tilde{\sigma}^{j}\{\mathcal{H}(\boldsymbol{\sigma}), \mathcal{H}(\tilde{\boldsymbol{\sigma}})\}_{P B} \\
& =\iint d^{5} \sigma d^{5} \tilde{\sigma} \sigma^{i} \tilde{\sigma}^{j}\left[M^{k}(\boldsymbol{\sigma})+M^{k}(\tilde{\boldsymbol{\sigma}})\right] \partial_{k} \delta^{5}(\boldsymbol{\sigma}-\tilde{\boldsymbol{\sigma}}) \\
& =\int d^{5} \sigma\left(\sigma^{i} M^{j}-\sigma^{j} M^{i}\right),
\end{aligned}
$$

where $\boldsymbol{M}=(\mathbb{H} \times \mathbb{H})$. Since $\mathbb{H} \times \mathbb{H}=\mathbb{B} \times \mathbb{B}$ whenever $\mathbf{L}$ is a conserved charge, we have

$$
\left\{L^{i}, L^{j}\right\}_{P B}=-J^{i j},
$$

as expected for a Lorentz boost.

We now aim to determine the implications of the Lorentz invariance condition (2.64) for the Hamiltonian density. To this end it is convenient to use the $\mathrm{SO}(5)$ rotational invariance to bring $\mathbb{B}$ to a skew-diagonal form (at any chosen spacetime point); we then have

$$
B_{12}=-B_{21}=b_{1}, \quad B_{34}=-B_{43}=b_{2} .
$$

This tells us that there are only two independent rotational scalars that we can construct from $\mathbb{B}$, and we may take them to be

$$
\begin{aligned}
s & =\frac{1}{2}|\mathbb{B}|^{2} \equiv \frac{1}{4} B^{i j} B^{k l} \delta_{i k} \delta_{j l}, \\
p & =|\mathbb{B} \times \mathbb{B}| \equiv \sqrt{(\mathbb{B} \times \mathbb{B}) \cdot(\mathbb{B} \times \mathbb{B})} .
\end{aligned}
$$

In terms of the skew-eigenvalues of $\mathbb{B}$, we have

$$
s=\frac{1}{2}\left(b_{1}^{2}+b_{2}^{2}\right), \quad p=\left|b_{1} b_{2}\right| .
$$

If we choose $\mathcal{H}$ to be a function of these two rotation scalars then $\mathbb{H}$ has components

$$
H_{i j}=\left(\mathcal{H}_{s}+2 s p^{-1} \mathcal{H}_{p}\right) B_{i j}+p^{-1} \mathcal{H}_{p}\left(B^{3}\right)_{i j} .
$$


As expected, we have $\mathbb{H}=\mathbb{B}$ for the free-field Hamiltonian density $\mathcal{H}=s$. We also have

$$
\mathbb{H} \times \mathbb{H}=\left[\mathcal{H}_{s}^{2}+2 s p^{-1} \mathcal{H}_{s} \mathcal{H}_{p}+\mathcal{H}_{p}^{2}\right](\mathbb{B} \times \mathbb{B}) .
$$

The condition (2.64) for Lorentz invariance is therefore equivalent to

$$
\mathcal{H}_{s}^{2}+\frac{2 s}{p} \mathcal{H}_{s} \mathcal{H}_{p}+\mathcal{H}_{p}^{2}=1
$$

This is formally identical to the equation (2.15) required for Lorentz invariance of the generic $4 \mathrm{D}$ electrodynamics, and this is why we have used the same notation.

We have already observed in the $4 \mathrm{D}$ context that the condition (2.72) takes the simpler form

$$
\mathcal{H}_{\mathrm{u}} \mathcal{H}_{\mathrm{v}}=1
$$

in terms of the new variables

$$
\mathrm{u}=\frac{1}{2}\left(s+\sqrt{s^{2}-p^{2}}\right), \quad \mathrm{v}=\frac{1}{2}\left(s-\sqrt{s^{2}-p^{2}}\right),
$$

but these variables are now $\mathrm{SO}(5)$-invariant rotation scalars; they remain well defined in this context because it follows from (2.69) that

$$
s^{2}-p^{2}=\frac{1}{4}\left(b_{1}^{2}-b_{2}^{2}\right)^{2} \geq 0 .
$$

The solution of (2.73) that previously led to the BI Hamiltonian density of (2.23) now yields the following Hamiltonian density for a chiral 2-form electrodynamics:

$$
\mathcal{H}=\sqrt{T^{2}+T|\mathbb{B}|^{2}+|\mathbb{B} \times \mathbb{B}|^{2}}-T .
$$

This is the Hamiltonian density for the chiral 2-form on the 6D Minkowski worldvolume of a static planar M5-brane in an 11D Minkowski vacuum [6, 13]. Its $T \rightarrow \infty$ limit is the free theory of Henneaux-Teitelboim [12] but the $T \rightarrow 0$ limit yields

$$
\mathcal{H}_{T=0}=|\mathbb{B} \times \mathbb{B}| .
$$

This defines the interacting conformal $6 \mathrm{D}$ chiral 2-form theory of $[5,6]$ which is a $6 \mathrm{D}$ analog of the $4 \mathrm{D}$ BB electrodynamics.

\subsubsection{Reduction/truncation to $4 \mathrm{D}$}

The correspondence just established between $6 \mathrm{D}$ chiral 2-form electrodynamics and 4D duality invariant electrodynamics can be understood very directly from the existence of a dimensional-reduction/truncation that takes any given 6D example into its corresponding $4 \mathrm{D}$ example. The dimensional reduction step proceeds by writing the 5 -space coordinates as $\left\{\sigma^{\alpha}, \sigma^{4}, \sigma^{5} ; \alpha=1,2,3\right\}$ and taking all fields to depend only on the $\sigma^{\alpha}$. In this case, one finds that

$$
\begin{array}{rlrl}
B^{\alpha \beta} & =\varepsilon^{\alpha \beta \gamma} \partial_{\gamma} A_{45}, & B^{45}=\frac{1}{2} \varepsilon^{\alpha \beta \gamma} \partial_{\alpha} A_{\beta \gamma}, \\
B^{\alpha 4}=\varepsilon^{\alpha \beta \gamma} \partial_{\beta} \tilde{A}_{\gamma}:=D^{\alpha}, & B^{\alpha 5}=\varepsilon^{\alpha \beta \gamma} \partial_{\beta} A_{\gamma}:=B^{\alpha},
\end{array}
$$


where

$$
A_{\alpha}=-A_{\alpha 4}, \quad \tilde{A}_{\alpha}=A_{\alpha 5} .
$$

The $6 \mathrm{D}$ rotation scalars $s$ and $p^{2}$ may now be written as

$$
\begin{aligned}
s \equiv \frac{1}{2}|\mathbb{B}|^{2} & =\frac{1}{2}\left(|\mathbf{D}|^{2}+|\mathbf{B}|^{2}\right)+\frac{1}{2}\left[\left(B^{45}\right)^{2}+\frac{1}{2} B_{\alpha \beta} B^{\alpha \beta}\right] \\
p^{2} \equiv|\mathbb{B} \times \mathbb{B}|^{2} & =|\mathbf{D} \times \mathbf{B}|^{2}+\frac{1}{8}\left(B^{45}\right)^{2} B^{\alpha \beta} B_{\alpha \beta} .
\end{aligned}
$$

As these expressions are at least quadratic in the variables $\left(A_{45}, A_{\alpha \beta}\right)$, the truncation

$$
A_{45}=0, \quad A_{\alpha \beta}=0,
$$

is a consistent one in the sense that the full field equations with $A_{45}, A_{\alpha \beta}$ set to zero are equivalent to the equations obtained from the truncated action.

After this truncation we have

$$
s \rightarrow \frac{1}{2}\left(|\mathbf{D}|^{2}+|\mathbf{B}|^{2}\right), \quad p \rightarrow|\mathbf{D} \times \mathbf{B}|,
$$

which are the expressions for the $4 \mathrm{D}$ rotation scalars $(s, p)$. The $6 \mathrm{D}$ Hamiltonian density therefore becomes the 4D Hamiltonian density of a duality-invariant 1-form electrodynamics. In addition,

$$
\frac{1}{4} \dot{A}_{i j} B^{i j} \rightarrow-\dot{\mathbf{A}} \cdot \nabla \times \tilde{\mathbf{A}}+\text { total derivative },
$$

which means that the $4 \mathrm{D}$ action is (2.39), which we showed to be equivalent to the manifestly duality-invariant action (2.48). The off-shell duality invariance of the action obtained from the $6 \mathrm{D}$ Hamiltonian formulation is guaranteed by the fact that the electromagnetic duality group is the $\mathrm{SO}(2)$ factor of the $\mathrm{SO}(3) \times \mathrm{SO}(2)$ subgroup of the $\mathrm{SO}(5)$ rotation group preserved by the reduction/truncation.

\subsection{D chiral 2-form: Perry-Schwarz formulation}

Although there is no standard Lagrangian formulation of chiral 2-form electrodynamics, there is an alternative canonical-type formulation in which the manifest symmetry is $5 \mathrm{D}$ Lorentz invariance. Let us take the 6D Minkowski metric to be

$$
d s_{6}^{2}=\eta_{m n}^{(5)} d x^{m} d x^{n}-(d y)^{2} \quad(m=0,1,2,3,4),
$$

where $\eta^{(5)}=\operatorname{diag}(1,-1,-1,-1,-1)$. Let $A_{m n}$ be the $5 \mathrm{D}$ components of the $6 \mathrm{D} 2$-form potential, and define

$$
B^{m n}=\frac{1}{2} \varepsilon^{m n p q r} \partial_{p} A_{q r} .
$$

All fields still depend on $y$ in addition to the 5D Minkowski coordinates, and the generic Perry-Schwarz action for them takes the form [15]

$$
I_{P S}=\int d y \int d^{5} x\left\{\frac{1}{4}\left(\partial_{y} A_{m n}\right) B^{m n}-\mathcal{V}\right\},
$$


where $\mathcal{V}$ is a 5D Lorentz-scalar function of $B$. This is a kind of phase-space action in which the role of time is played by the space coordinate $y$, with field equations that are first-order in $\partial_{y}$ rather that $\partial_{t}$.

One possible basis for 5D Lorentz scalars is

$$
Q=\frac{1}{4} B^{m n} B_{m n}, \quad R=\sqrt{Q^{2}-w^{m} w_{m}},
$$

where

$$
w_{m}=\frac{1}{8} \varepsilon_{m n p q r} B^{n p} B^{q r},
$$

and the 5D Lorentz indices are raised or lowered with the 5D Minkowski metric; a useful identity (which is a consequence of the obvious identity $B^{[m n} B^{p q} B^{r s]} \equiv 0$ ) is

$$
w_{m} B^{m n} \equiv 0 \quad(n=0,1,2,3,4) .
$$

The variable $R$ is manifestly real when the one-form $w$ is spacelike. To see that this is still true when $w$ is timelike we observe that in this case we may choose coordinates such that only $w_{0}$ is non-zero; the identity (2.89) then implies that $B^{0 i}=0(i=1,2,3,4)$ from which it follows that

$$
Q=-\frac{1}{4} \operatorname{tr} \mathbb{B}^{2}, \quad w_{0}^{2}=\frac{1}{4}\left[\operatorname{tr} \mathbb{B}^{4}-\frac{1}{2}\left(\operatorname{tr} \mathbb{B}^{2}\right)^{2}\right],
$$

where (as in the previous subsection) $\mathbb{B}$ is the $4 \times 4$ matrix with entries $B^{i j}$. We then have

$$
Q^{2}-w^{m} w_{m}=\frac{1}{16}\left(\operatorname{tr} \mathbb{B}^{2}\right)^{2}-w_{0}^{2}=\frac{1}{4} \operatorname{tr}\left[\mathbb{B}^{2}-\frac{1}{4}\left(\operatorname{tr} \mathbb{B}^{2}\right) \mathbb{I}_{4}\right]^{2} \geq 0 .
$$

The condition on $\mathcal{V}$ for 6D Lorentz invariance was found in [15] using a different basis of $5 \mathrm{D}$ Lorentz scalars; the equivalent equation for $\mathcal{V}(Q, R)$ is

$$
\mathcal{V}_{Q}^{2}-\mathcal{V}_{R}^{2}=1
$$

In terms of the new variables

$$
U=\frac{1}{2}(Q-R), \quad V=\frac{1}{2}(Q+R),
$$

the equation for $6 \mathrm{D}$ Lorentz invariance is [15]

$$
\mathcal{V}_{U} \mathcal{V}_{V}=1
$$

This equation is formally equivalent to the equation (2.22) for the Lorentz invariant Lagrangian of a generic non-linear 4D electrodynamics to have EL equations that are invariant under an $\mathrm{SO}(2)$ electromagnetic duality. As we now explain, this is not a coincidence.

\subsubsection{D reduction redux}

If we dimensionally reduce the generic Perry-Schwarz action of (2.86) from 6D to 5D by setting

$$
\partial_{y} A_{m n}=0
$$


then we have a manifestly Lorentz invariant 5D theory with Lagrangian density

$$
\mathcal{L}_{5 D}=-\mathcal{V}
$$

Let $\left\{x^{\mu}, x^{4} ; \mu=0,1,2,3\right\}$ be the 5D Minkowski coordinates, and let us dimensionally reduce/truncate to $4 \mathrm{D}$ by setting

$$
\partial_{4} A_{\mu 4}=0, \quad A_{\mu \nu}=0 .
$$

Then the only non-zero components of $B$ are

$$
B^{\mu \nu}=\varepsilon^{\mu \nu \rho \sigma} \partial_{\rho} A_{\sigma 4}=\frac{1}{2} \varepsilon^{\mu \nu \rho \sigma} F_{\rho \sigma},
$$

where $F=d A$ for $A=d x^{\mu} A_{\mu 4}$, and the only non-zero component of $w$ is

$$
w_{4}=\frac{1}{8} \varepsilon_{\mu \nu \rho \sigma} B^{\mu \nu} B^{\rho \sigma}=-\frac{1}{8} \varepsilon^{\mu \nu \rho \sigma} F_{\mu \nu} F_{\rho \sigma} .
$$

We now find that

$$
Q=S, \quad R=\sqrt{S^{2}+P^{2}},
$$

where $(S, P)$ are the standard $4 \mathrm{D}$ Lorentz scalars. It follows that $(U, V)$ of $(2.93)$ are now the $4 \mathrm{D}$ variables introduced in (2.21), so the Perry-Schwarz equation (2.94) for $6 \mathrm{D}$ Lorentz invariance of the generic 6D chiral 2-form electrodynamics (with 5D Lorentz invariance manifest) reduces to the Gibbons-Rasheed equation (2.22) for electromagnetic duality invariance of the generic 4D 1-form electrodynamics, with Lagrangian density

$$
\mathcal{L}_{4 D}=-\mathcal{V}(S, P)
$$

\section{PST formulation}

There exist several approaches to the construction of manifestly Lorentz-invariant dualitysymmetric or chiral $p$-form actions; they all use additional fields of some kind (e.g. [11, 16, $17,39-52])$. The PST formulation $[16,17]$ is particularly economical, since it uses a single auxiliary scalar field or, more precisely, a nowhere-null closed one-form. The covariant PST construction allows for a straightforward coupling of chiral $p$-forms to gravity. In addition, it connects different non-manifestly Lorentz-invariant formulations [18, 53, 54] (such as [55] to [36] and [12] to [15]) and has led to some novel results; an example is an M5-brane action $[56,57]$ of a type relevant to a more general construction that we discuss in this section and in sections 5 and 6 .

The PST formulation of chiral $2 n$-form electrodynamics in $D=4 n+2$ dimensions starts from a potential $2 n$-form $A$ on spacetime with $H=d A$ its $(2 n+1)$-form fieldstrength, and a closed spacetime one form $v$, which may be timelike or spacelike. For the Minkowski spacetime background that we assume here, we have $v=d a$ for scalar field $a$, and a PST gauge invariance that allows us to identify $a$ with the Minkowski time coordinate (if $v$ is timelike) or with a Minkowski space coordinate (if $v$ is spacelike). These two versions of the PST actions are off-shell inequivalent (i.e. not related by local field 
redefinitions or gauge transformations) but they are expected to be on-shell equivalent (i.e. have equivalent field equations) for the following reason: ${ }^{5}$ the PST field equation reduces to a manifestly Lorentz invariant non-linear self-duality condition that involves $v$, but one expects to be able to rewrite this in a form that is both manifestly Lorentz invariant and $v$-independent because there is no obstacle to manifest Lorentz covariance at the level of field equations. This is known to be true for the linear chiral $p$-form theories and has been proved for the M5-brane equations, with the help of their interpretation as Lorentz covariant superembeddings [60-63].

We focus in this section on the $6 \mathrm{D}$ chiral 2-form theories. We choose standard Minkowski coordinates $\left\{x^{M} ; M=0,1, \ldots, 5\right\}$ for the $6 \mathrm{D}$ Minkowski background, with a metric of 'mostly minus' signature. From the components $H_{M N P}$ of $H$, and $v_{M}$ of $v$, we construct the gauge-invariant anti-symmetric tensor density

$$
B^{M N}=-\frac{1}{6} \varepsilon^{M N P Q R S} H_{P Q R} v_{S}, \quad\left(v_{S}=\partial_{S} a\right) .
$$

The general $6 D$ chiral 2-form PST action has the following form:

$$
S=\int d^{6} x\left(\frac{1}{4 v^{2}} B^{M N} H_{M N P} v^{P}-\mathcal{V}\right), \quad\left(v^{2}=v^{S} v_{S}\right)
$$

where $\mathcal{V}$ is a function of the two Lorentz scalars

$$
Q_{1}=-\frac{1}{4 v^{2}} B^{M N} B_{M N}, \quad Q_{2}=Q_{1}^{2}+\frac{1}{v^{2}} w^{M} w_{M}
$$

with

$$
w_{M}=-\frac{1}{8 v^{2}} \varepsilon_{M N P Q R S} B^{N P} B^{Q R} v^{S} .
$$

Notice that $v_{M} w^{M} \equiv 0$, so $w$ is non-timelike if $v$ is timelike, but is unrestricted if $v$ is spacelike. The variable $Q_{2}$ is manifestly non-negative unless either (i) $v$ is timelike and $w$ spacelike, or (ii) $v$ is spacelike and $w$ timelike. However, in these cases we may construct the $6 \times 6$ projector matrix

$$
\mathrm{P}^{M}{ }_{N}=\delta^{M}{ }_{N}-\frac{v^{M} v_{N}}{v^{2}}-\frac{w^{M} w_{N}}{w^{2}} .
$$

A calculation similar to the one leading to (2.91) now yields

$$
\begin{aligned}
Q_{1}^{2}+\frac{1}{v^{2}} w^{M} w_{M} & =\frac{1}{4\left(v^{2}\right)^{2}}\left[\operatorname{tr} B^{4}-\frac{1}{4}\left(\operatorname{tr} B^{2}\right)^{2}\right] \\
& =\frac{1}{4\left(v^{2}\right)^{2}} \operatorname{tr}\left[B^{2}-\frac{1}{4}\left(\operatorname{tr} B^{2}\right) \mathrm{P}\right]^{2} \geq 0
\end{aligned}
$$

where the second equality relies on the following properties of P:

$$
\operatorname{tr} \mathrm{P}=4, \quad(\mathrm{P} B)^{M}{ }_{N}=B^{M}{ }_{N} .
$$

\footnotetext{
${ }^{5}$ This may not apply in non-Minkowski backgrounds because of topological issues in the spacelike- $v$ PST formulation $[54,58,59]$, and in some cases that will be discussed later.
} 
The second of these properties is a consequence of the identity $v_{M} B^{M N} \equiv 0$, because (i) this implies that $\operatorname{det} B=0$, and (ii)

$$
w_{M} B^{M N} \propto v^{N} \sqrt{\operatorname{det} B}
$$

which is a consequence of the Schouten identity ${ }^{6}$

$$
v^{[T} B^{P Q} B^{R S} B^{M] N} \equiv 6 v^{N} B^{[P Q} B^{R S} B^{M T]} .
$$

This concludes the proof that $Q_{2} \geq 0$ in all cases.

Because the PST Lagrangian density (3.2) depends on the scalar field $a$ in addition to the chiral 2-form fields, equivalence to the Hamiltonian or Perry-Schwarz formulations depends upon the possibility of a PST gauge invariance that will allow $a$ to be set to some fixed function on spacetime. The infinitesimal form of the PST gauge transformations that allows this is

$$
\delta_{\varphi} a=\varphi(x), \quad \delta_{\varphi} A=\frac{\varphi(x)}{v^{2}}\left(i_{v} H_{3}-\mathcal{V}_{B}\right)
$$

where $\mathcal{V}_{B}$ is the two form with components $\partial \mathcal{V} / \partial B^{M N}$. However, this is a gauge invariance of the PST action with Lagrangian density (3.2) only if $\mathcal{V}$ satisfies [9]

$$
\left(\frac{\partial \mathcal{V}}{\partial Q_{1}}\right)^{2}-4 Q_{2}\left(\frac{\partial \mathcal{V}}{\partial Q_{2}}\right)^{2}=1
$$

We shall give more details about properties of the PST formulation (in particular the form of the PST field equations) in sections 5 and 6 . Here we explain how the Hamiltonian and Perry-Schwarz formulations are recovered from the PST action by gauge fixing.

\subsection{Timelike $v$}

If $v$ is timelike then the PST gauge invariance allows us to choose $a=t \equiv x^{0}$. The only non-zero components of $B$ and $w$ are now $\mathbb{B}$ and $\mathbb{B} \times \mathbb{B}$, respectively, where $\mathbb{B}$ is the 5 -space antisymmetric tensor density of subsection 2.3, and the PST action becomes that of (2.54) with $\mathcal{V}=\mathcal{H}$. After making this PST gauge choice we also find that

$$
Q_{1}=-s, \quad Q_{2}=s^{2}-p^{2},
$$

where $(s, p)$ are the $6 \mathrm{D}$ rotation scalars defined in $(2.68)$. When written in terms of $(s, p)$ the condition (3.11) on $\mathcal{V}$ is identical to (2.72), which is the condition for the Hamiltonian density $\mathcal{H}(s, p)$ to define a $(6 \mathrm{D})$ Lorentz invariant theory.

To summarize, the PST action (3.2) for timelike $v$ is a 'covariant' version of the Hamiltonian phase-space action; by using (3.12) to rewrite (3.11) as a PDE for $\mathcal{H}(s, p)=$ $\mathcal{V}\left(Q_{1}, Q_{2}\right)$ one recovers the PDE (2.72).

\footnotetext{
${ }^{6}$ That is, an identity equivalent to the obvious over-antisymmetrization identity on seven indices.
} 


\subsubsection{D reduction/truncation prior to gauge-fixing of $v$}

We have already seen that the phase-space action for the generic $4 \mathrm{D}$ Lorentz and duality invariant 1-form electrodynamics may be found by a reduction/truncation of the phase-space action for the generic 6D chiral 2-form electrodynamics. An analogous truncation/reduction may be carried out directly on the 6D PST action with timelike $v$, prior to gauge fixing. This yields the 4D PST action of [64], which reduces to the phase-space action (2.48) upon PST gauge fixing. Prior to this gauge fixing, both Lorentz and duality invariance are manifest. We present a brief summary of this formulation of $4 \mathrm{D}$ nonlinear electrodynamics theories, as we shall be using its higher-dimensional extension [8] in section 5 .

The duality doublets of 3-vector 'electric' and 'magnetic' fields of (2.48) are replaced by duality doublets of the 4-vector 'electric' and 'magnetic' fields

$$
E_{\mu}^{\mathrm{a}}=F_{\mu \nu}^{\mathrm{a}} \hat{v}^{\nu}, \quad B_{\mu}^{\mathrm{a}}=\tilde{F}_{\mu \nu}^{\mathrm{a}} \hat{v}^{\nu},
$$

where $F^{\mathrm{a}}=d A^{\mathrm{a}}$ and $\tilde{F}^{\mathrm{a}}$ its Hodge dual, and

$$
\hat{v}=v / \sqrt{v^{2}}, \quad v=d a .
$$

The action for these fields is ${ }^{7}$

$$
I\left[A^{1}, A^{2}\right]=\int d^{4} x\left\{\frac{1}{2} \varepsilon_{\mathrm{ab}} E^{\mathrm{a}} \cdot B^{\mathrm{b}}-\mathcal{H}\left(B^{1}, B^{2}\right)\right\},
$$

where

$$
E^{\mathrm{a}} \cdot B^{\mathrm{b}}=E_{\mu}^{\mathrm{a}} B^{\mathrm{b} \mu}=E_{0}^{\mathrm{a}} B_{0}^{\mathrm{b}}-\sum_{i=1}^{3} E_{i}^{\mathrm{a}} B_{i}^{\mathrm{b}} .
$$

This action is invariant under the gauge transformation [16]

$$
\delta A^{\mathrm{a}}=v \phi^{\mathrm{a}}
$$

where $\phi^{\mathrm{a}}$ is a duality doublet of scalar parameters. It is also invariant under the (PST) gauge transformation

$$
\delta a=\varphi(x), \quad \delta A^{\mathrm{a}}=-\frac{\varphi(x)}{\sqrt{v^{2}}}\left(E^{\mathrm{a}}-\varepsilon^{\mathrm{ab}} \frac{\partial \mathcal{H}}{\partial B^{\mathrm{b}}}\right)
$$

for an arbitrary function $\varphi$. This allows the gauge choice $a=t$, which reduces the action to (2.48), as claimed. ${ }^{8}$ It also reduces the gauge transformation (3.17) to the gauge transformation (2.49) that leaves invariant the action (2.48).

If $\mathcal{H}$ is $\mathrm{SO}(2)$-duality invariant then it must be some function of the duality-invariant Lorentz scalars

$$
s=-\frac{1}{2} B^{\mathrm{a}} \cdot B^{\mathrm{a}}, \quad p=\sqrt{q},
$$

\footnotetext{
${ }^{7}$ An example is the BI theory on the D3-brane considered in $[1,2]$.

${ }^{8}$ In verifying this, and statements below, it should be remembered that we use a Lorentz metric with 'mostly-minus' signature, as in (3.16).
} 
where $q$ is the $S l(2 ; \mathbb{R})$-duality invariant

$$
q=\frac{1}{2} \epsilon_{\mathrm{ac}} \epsilon_{\mathrm{bd}}\left(B^{\mathrm{a}} \cdot B^{\mathrm{b}}\right)\left(B^{\mathrm{c}} \cdot B^{\mathrm{d}}\right) .
$$

The notation is motivated by the fact that in the PST gauge $v=d t$ we find, using the definitions of $\mathbf{B}^{\mathrm{a}}$ in (2.44), that

$$
s \rightarrow \frac{1}{2}\left(|\mathbf{D}|^{2}+|\mathbf{B}|^{2}\right), \quad p \rightarrow|\mathbf{D} \times \mathbf{B}|,
$$

which are the independent rotation scalars introduced in subsection 2.2. As we saw for the $6 \mathrm{D}$ case, the PDE (2.15) that is required for Lorentz invariance of the $4 \mathrm{D}$ phase-space action with Hamiltonian density $\mathcal{H}(s, p)$ becomes the condition for PST gauge-invariance of the PST action (3.15) when $(s, p)$ are re-interpreted as the duality-invariant Lorentz scalars (3.19). When rewritten as a PDE for $\mathcal{H}(s, q)$, this condition is

$$
\mathcal{H}_{s}^{2}+4 s \mathcal{H}_{s} \mathcal{H}_{q}+4 q \mathcal{H}_{q}^{2}=1
$$

\subsection{Spacelike $v$}

When $v$ is spacelike the PST gauge invariance allows us to set $a=y \equiv x^{5}$. For this PST gauge choice the only non-zero components of $B$ and $w$ are

$$
B^{m n}=\frac{1}{2} \varepsilon^{m n p q r} \partial_{p} A_{q r}, \quad w_{m}=-\frac{1}{8} \varepsilon_{m n p q r} B^{n p} B^{q r},
$$

and the Lagrangian density (3.2) becomes that of the Perry-Schwarz action (2.86). Also, the variables $\left(Q_{1}, Q_{2}\right)$ on which $\mathcal{V}$ depends are now

$$
Q_{1}=\frac{1}{4} B^{m n} B_{m n}=Q, \quad Q_{2}=Q^{2}-w^{m} w_{m}=R^{2},
$$

where $(Q, R)$ are the variables introduced in (2.87). Using this result to rewrite (3.11) as an equation for $\mathcal{V}(Q, R)$ we recover (2.92).

To summarize, the PST action for spacelike $v$ is a 'covariant' version of the PerrySchwarz action. Hence, its $4 D$ dimensional reduction/truncation with $v=d x^{5}$ yields the $4 D$ Lagrangian (2.101).

\subsection{Variant PST formulations}

In addition to the formulation which uses the one form $v=d a$ considered above, there are other variants of the PST action. For instance, in $D=6$ one can dualize the (timelike) one-form $v=d a$ to a 5 -form field-strength whose Hodge dual is a nowhere-zero spacelike one-form $u$; this yields a 'dual' PST formulation [53, 54]. Technically, this procedure amounts to performing the following replacements in the PST Lagrangian (3.2): $B_{M N} \rightarrow H_{M N L} u^{L}$ and $H_{M N L} v^{L} \rightarrow-B_{M N}$ with the timelike $v$ replaced by the spacelike $u$. In this case the reduction/truncation to $4 \mathrm{D}$ yields, on setting $u=d x^{5}$, the Lagrangian density of (2.101). In contrast, for the gauge choice $u=d x$ for a non-compact coordinate $x$, reduction/truncation yields a non-linear generalization of the duality-symmetric 
electrodynamics of Zwanziger [55]. An example is the dual manifestly duality-symmetric Born-Infeld-like action on the D3-brane $[65,66]$ which can be found by dimensional reduction/truncation of a corresponding variant of the M5-brane action [54, 67]. Yet another form of the M5-brane action, with a triplet of auxiliary closed one-forms, was constructed in [68]. It is related to an effective gauge field theory for multiple M2-branes with volume preserving diffeomorphisms of an 'internal' 3-manifold as its gauge group [69-73].

\section{New examples in $4 \mathrm{D}$ and $6 \mathrm{D}$}

So far, we have exhibited a correspondence between any given Lorentz-invariant 6D theory of chiral 2-form electrodynamics and an associated Lorentz and duality invariant 4D theory of 1-form electrodynamics. Within either the Hamiltonian or Lagrangian formulation, the correspondence comes about because the Hamiltonian/Lagrangian density is a function of two variables subject to a non-linear first-order PDE, which is formally the same in both cases for a particular choice of bases for the two sets of two independent variables. Moreover, the basis of independent variables can be chosen such that the PDE is also formally the same for both the Hamiltonian and Lagrangian formulations (if, for $6 \mathrm{D}$, we view as "Lagrangian" the formulation of [15] with manifest 5D Lorentz invariance). Thus, remarkably, the task of constructing these $4 \mathrm{D} / 6 \mathrm{D}$ theories reduces to the solution of a single PDE for a function of two variables, where only the interpretation of variables distinguishes between 4D/6D and Hamiltonian/Lagrangian.

One form of this 'universal' PDE is (2.73) where the dependent variable is the Hamiltonian density and the independent variables $(\mathrm{u}, \mathrm{v})$ are $(4 \mathrm{D}$ or $6 \mathrm{D})$ rotation scalars. We have already discussed the solution that yields Born-Infeld electrodynamics in the $4 \mathrm{D}$ context; as it applies equally in 6D, and as both have brane interpretations within String/M-theory, we shall call it the 'D3/M5' solution.

Here we focus on the one-parameter extension of the D3/M5 solution that we found recently in the context of $4 \mathrm{D}$ electrodynamics [3] by considering a Hamiltonian density of the form

$$
\mathcal{H}(\mathrm{u}, \mathrm{v})=\sqrt{K(\mathrm{u}, \mathrm{v})}+\text { constant } .
$$

The 'universal' PDE (2.73) satisfied by $\mathcal{H}$ becomes the following PDE for $K$ :

$$
K_{\mathrm{u}} K_{\mathrm{v}}=4 K
$$

An obvious ansatz for $K$ is the generic quadratic function

$$
K=c+b_{1} \mathrm{u}+b_{2} \mathrm{v}+a_{11} \mathrm{u}^{2}+2 a_{12} \mathrm{uv}+a_{22} \mathrm{v}^{2} .
$$

This is a solution of (4.2) provided that

$$
a_{11}\left(a_{12}-1\right)=0, \quad a_{22}\left(a_{12}-1\right)=0, \quad a_{11} a_{22}+a_{12}^{2}=2 a_{12},
$$

and

$$
\left(a_{12}-2\right) b_{1}+a_{11} b_{2}=0, \quad\left(a_{12}-2\right) b_{2}+a_{22} b_{1}=0, \quad b_{1} b_{2}=4 c .
$$


There are two types of solution of these algebraic relations, according to whether we choose (i) $a_{12}=1$ or (ii) $a_{12}=2$, in which case $a_{11}=a_{22}=0$. For case (i), $K$ is a perfect square and this leads to a Hamiltonian density linear in $(\mathrm{u}, \mathrm{v})$ that can be recovered as the weakfield limit of the Hamiltonian density resulting from case (ii). The algebraic relations for case (ii) determine $K$ in terms of a constant $T$ with dimensions of energy density, and a dimensionless parameter $\gamma$. Requiring $\mathcal{H}$ to be real for all values of $(\mathrm{u}, \mathrm{v})$ fixes the sign of $T$, and requiring it to be zero in the vacuum fixes the arbitrary additive constant. This results in the Hamiltonian density

$$
\mathcal{H}=\sqrt{T^{2}+2 T\left(e^{-\gamma} \mathbf{u}+e^{\gamma} \mathrm{v}\right)+4 \mathrm{uv}}-T,
$$

where $\gamma \in \mathbb{R}$ is a numerical parameter.

For $\gamma=0$ we recover the D3/M5 result. The strong-field $(T \rightarrow 0)$ limit $\mathcal{H}=2 \sqrt{\mathrm{uv}}$ is the same as that of the D3/M5 solution, see (2.25), but the weak-field $(T \rightarrow \infty)$ limit still involves the parameter $\gamma$ :

$$
\begin{aligned}
\left.\mathcal{H}\right|_{T=\infty} & =e^{-\gamma} \mathbf{u}+e^{\gamma} \mathbf{v} \\
& =(\cosh \gamma) s-(\sinh \gamma) \sqrt{s^{2}-p^{2}}
\end{aligned}
$$

This Hamiltonian density is manifestly non-negative; it is always real since (as we have already seen) $s^{2}-p^{2} \geq 0$ for either the $4 \mathrm{D}$ or $6 \mathrm{D}$ interpretation of the variables $(s, p)$. The $4 \mathrm{D}$ interpretation yields an interacting extension of Maxwell's equations that preserves both electromagnetic duality and conformal invariance [3]. We briefly review this result below, and then consider the analogous $6 \mathrm{D}$ chiral 2 -form electrodynamics.

\subsection{ModMax electrodynamics}

Using the $4 \mathrm{D}$ interpretation for the parameters $(\mathrm{u}, \mathrm{v})$, and their relation to the rotation scalars $(s, p)$, the Hamiltonian density of $(4.6)$ is found to be

$$
\mathcal{H}_{(T)}=\sqrt{T^{2}+2 T\left[(\cosh \gamma) s-(\sinh \gamma) \sqrt{s^{2}-p^{2}}\right]+p^{2}}-T .
$$

This is the one-parameter generalization of the BI Hamiltonian density found in [3]. Its strong-field $(T \rightarrow 0)$ limit yields the Hamiltonian density (2.25) of Bialynicki-Birula electrodynamics. Its weak-field $(T \rightarrow \infty)$ limit is $(4.7)$ with the $4 \mathrm{D}$ interpretation of $(s, p)$; i.e.

$$
\mathcal{H}=\frac{1}{2}(\cosh \gamma)\left(|\mathbf{D}|^{2}+|\mathbf{B}|^{2}\right)-\frac{1}{2}(\sinh \gamma) \sqrt{\left(|\mathbf{D}|^{2}+|\mathbf{B}|^{2}\right)^{2}-4|\mathbf{D} \times \mathbf{B}|^{2}} .
$$

The Maxwell Hamiltonian density is recovered for $\gamma=0$, while for $\gamma>0$ one gets the oneparameter modification of Maxwell electrodynamics called "ModMax" electrodynamics in [3]. The ModMax Hamiltonian field equations are

$$
\dot{\mathbf{B}}=-\nabla \times\left[\mathcal{A}_{-} \mathbf{D}-\mathcal{C} \mathbf{B}\right], \quad \dot{\mathbf{D}}=\nabla \times\left[\mathcal{A}_{+} \mathbf{B}-\mathcal{C} \mathbf{D}\right] .
$$

for coefficient functions

$$
\mathcal{A}_{ \pm}=\cosh \gamma \pm \sinh \gamma \cos \Theta, \quad \mathcal{C}=\sinh \gamma \sin \Theta,
$$


where the angular variable $\Theta$ is most simply defined in terms of the rotation scalars $(\xi, \eta)$ of (2.11) by

$$
(\xi, \eta)=\sqrt{\eta^{2}+\xi^{2}}(\cos \Theta, \sin \Theta) .
$$

The Legendre transform of $\mathcal{H}_{(T)}$ of (4.8) yields the Lagrangian density ${ }^{9}$

$$
\mathcal{L}_{(T)}=T-\sqrt{T^{2}-2 T\left[(\cosh \gamma) S+(\sinh \gamma) \sqrt{S^{2}+P^{2}}\right]-P^{2}} .
$$

This result is most easily found using methods explained in section 5. It reduces to the usual BI Lagrangian density for $\gamma=0$, and in the $T \rightarrow \infty$ limit it reduces to the ModMax Lagrangian density obtained in [3] by Legendre transform of the ModMax Hamiltonian density (4.9):

$$
\mathcal{L}=-\frac{1}{2}(\cosh \gamma)\left(|\mathbf{E}|^{2}-|\mathbf{B}|^{2}\right)+\frac{1}{2}(\sinh \gamma) \sqrt{\left(|\mathbf{E}|^{2}-|\mathbf{B}|^{2}\right)^{2}+4|\mathbf{E} \cdot \mathbf{B}|^{2}} .
$$

By construction, this Lagrangian defines a duality-invariant 4D electrodynamics, and one may verify that it satisfies the duality-invariance condition (2.20); see also [74].

We refer the reader to [3] for more details of properties of the ModMax theory, but we mention here that $\gamma \geq 0$ is necessary to eliminate the possibility of superluminal propagation of small-amplitude fluctuations about a background solution of constant electric/magnetic fields, and that there are exact Maxwell-like plane-wave solutions for $\gamma \geq 0$. See also [75-78] for recent studies of ModMax effects on self-gravitating solutions in General Relativity.

An alternative to using a Legendre transform to relate 4D Hamiltonian and Lagrangian densities, is to obtain both from an analogous $6 \mathrm{D}$ chiral 2-form theory by reduction/truncation, so we now turn to this $6 \mathrm{D}$ theory.

\subsection{New chiral 2-form theories}

Using the $6 \mathrm{D}$ interpretation for the parameters $(\mathrm{u}, \mathrm{v})$, and their relation to the $6 \mathrm{D}$ rotation scalars $(s, p)$, the Hamiltonian density (4.6) is formally the same as it was for 4 D, i.e. (4.8). Using (2.68); i.e. $s=\frac{1}{2}|\mathbb{B}|^{2}$ and $p=|\mathbb{B} \times \mathbb{B}|$, we arrive at the $6 \mathrm{D}$ Hamiltonian density

$$
\mathcal{H}=\sqrt{T^{2}+T\left[(\cosh \gamma)|\mathbb{B}|^{2}-(\sinh \gamma) \sqrt{|\mathbb{B}|^{4}-4|\mathbb{B} \times \mathbb{B}|^{2}}\right]+|\mathbb{B} \times \mathbb{B}|^{2}}-T .
$$

This reduces to $(2.76)$ for $\gamma=0$, and the strong-field $(T \rightarrow 0)$ limit is again (2.77) irrespective of the value of $\gamma$. However, the weak-field $(T \rightarrow \infty)$ limit is

$$
\mathcal{H}_{T=\infty}=\frac{1}{2}(\cosh \gamma)|\mathbb{B}|^{2}-\frac{1}{2}(\sinh \gamma) \sqrt{|\mathbb{B}|^{4}-4|\mathbb{B} \times \mathbb{B}|^{2}} .
$$

This defines a new interacting chiral 2-form electrodynamics; we shall show later that it is conformal invariant.

\footnotetext{
${ }^{9}$ This does not appear in [24] because only solutions of the PDE (2.20) that yield Maxwell electrodynamics in the weak-field limit were considered.
} 
The field equation is (2.56) (i.e. $\dot{\mathbb{B}}=\nabla \times \mathbb{H}$ ) and for the weak-field limit we have

$$
\mathbb{H}_{T=\infty}=\left[\cosh \gamma-(\sinh \gamma) \frac{s}{\sqrt{s^{2}-p^{2}}}\right] \mathbb{B}-(\sinh \gamma) \frac{p}{\sqrt{s^{2}-p^{2}}} \mathbf{n} \times \mathbb{B},
$$

where $\mathbf{n}$ is the unit 5 -vector in the direction defined by $-\mathbb{B} \times \mathbb{B}$, and

$$
[\mathbf{n} \times \mathbb{B}]^{i j}:=\frac{1}{2} \varepsilon^{i j k l m} n_{k} B_{l m} .
$$

The sign choice made for the unit 5 -vector $\mathbf{n}$ ensures that it becomes the unit 3 -vector of (2.26) after reduction/truncaton to 4D. As we saw earlier, this procedure replaces the $6 \mathrm{D}$ interpretation of the variables $(s, p)$ by their $4 \mathrm{D}$ interpretation, which means that the 6D Hamiltonian density (4.16) becomes the 4D ModMax Hamiltonian density of (4.9).

\subsubsection{PST formulation}

Let us return to the PST Lagrangian (3.2). The solution of (3.11) corresponding to (4.6) is

$$
\mathcal{V}_{(T)}=\sqrt{T^{2}-2 T\left[(\cosh \gamma) Q_{1}+(\sinh \gamma) \sqrt{Q_{2}}\right]+Q_{1}^{2}-Q_{2}}-T
$$

In the $v=d t$ gauge, for which $\left(Q_{1}, Q_{2}\right)$ are given in terms of the $\mathrm{SO}(5)$ rotation variables by $(3.12)$, and $\mathcal{V}=\mathcal{H}$, we may use $(2.74)$ to rewrite $\mathcal{V}_{(T)}$ in terms of $(\mathrm{u}, \mathrm{v})$; the result is precisely the Hamiltonian density of (4.6).

Besides the manifest Lorentz invariance, an advantage of the PST formulation is that we can also choose the $v=d x^{5}$ gauge to arrive at the Perry-Schwarz formulation of the same $6 \mathrm{D}$ field theory. In this case the reduction/truncation to $4 \mathrm{D}$ described in section 2.4 yields the Lagrangian density (4.13) for the generalized BI theory. This illustrates the fact that switching from timelike $v$ to spacelike $v$ in the PST formulation effects a Legendre transform of the $4 \mathrm{D}$ theory obtained by the reduction/truncation procedure described earlier.

\subsubsection{Conformal invariance of the weak/strong-field limits}

A feature of both the strong-field and weak-field limits of (4.19) is that there is no dependence on dimensionful parameters. This suggests that these limits yield conformal chiral 2-form electrodynamics theories, and this is already known to be true for the 'M5' case [5, 6]. Here we present a general proof of conformal invariance based on the observation of Zumino [79] that a theory defined for an arbitrary background spacetime metric is conformal invariant for a Minkowski background if its action for a general background depends on the background metric only through its conformal class; i.e. if it is Weyl invariant. ${ }^{10}$

A potential difficulty with this idea is that the generalization from Minkowski to generic spacetime metric could violate essential gauge invariances. This difficulty does not arise in our case because PST gauge invariance survives the coupling to gravity. In any case,

\footnotetext{
${ }^{10}$ This observation is a special case of a more general one [80]: Weyl invariance for a general background implies invariance under the diffeomorphisms generated by the conformal Killing vectors of any specific choice of background metric.
} 
Zumino's argument does not really depend on the generalization to an arbitrary background metric: it suffices to consider the metric of the Minkowski background in arbitrary coordinates. Once we have an action of coordinate independent form, which will be the case if it is the integral of a scalar density, then Weyl invariance implies that the action depends only on the conformal class of the background metric, and is therefore invariant under those diffeomorphisms generated by its conformal Killing vectors, whose algebra is the algebra of conformal isometries of the background (6D Minkowski in our case).

To apply this method we rewrite the PST Lagrangian density (3.1) in arbitrary coordinates, with $g$ as the Minkowski metric. The definition of $B$ in (3.1) is unchanged if we interpret $\varepsilon$ as the metric-independent alternating tensor density of unit weight defined (in any coordinate system) by $\varepsilon^{012345}=1$. If we also interpret $\mathcal{V}$ in $(3.2)$ as a scalar function of the scalars $\left(Q_{1}, Q_{2}\right)$ then the integrand of (3.2) becomes

$$
\mathcal{L}_{P S T}=\frac{1}{4 v^{2}} g^{P Q}\left(B^{M N} H_{M N P} v_{Q}\right)-\sqrt{|\operatorname{det} g|} \mathcal{V}
$$

where

$$
v^{2}=g^{M N} v_{M} v_{N} .
$$

Similarly, the scalars $\left(Q_{1}, Q_{2}\right)$ in arbitrary Minkowski coordinates are

$$
\begin{aligned}
Q_{1} & =-\frac{1}{4 v^{2}|\operatorname{det} g|} g_{M P} g_{N Q} B^{M N} B^{P Q} \\
Q_{2} & =Q_{1}^{2}+\frac{1}{v^{2}|\operatorname{det} g|} g^{M N} w_{M} w_{N}
\end{aligned}
$$

where $^{11}$

$$
w_{M}=-\frac{1}{8 v^{2}} g^{S T}\left(\varepsilon_{M N P Q R S} B^{N P} B^{Q R} v_{T}\right) .
$$

The factors of $1 /|\operatorname{det} g|$ ensure that $\left(Q_{1}, Q_{2}\right)$ are scalars rather than scalar densities.

Next, we consider the effect of the Weyl rescaling

$$
g \rightarrow \Omega^{2} g \quad\left(\Rightarrow \sqrt{|\operatorname{det} g|} \rightarrow \Omega^{6} \sqrt{|\operatorname{det} g|}\right) .
$$

This has no effect on the first term of (4.20), but it leads to the following rescaling of $Q_{1}$ and $\sqrt{Q_{2}}$ :

$$
Q_{1} \rightarrow \Omega^{-6} Q_{1}, \quad \sqrt{Q_{2}} \rightarrow \Omega^{-6} \sqrt{Q_{2}} .
$$

Notice that the factors of $\Omega^{-6}$ here are precisely what is needed to cancel the factor of $\Omega^{6}$ coming from the $\sqrt{|\operatorname{det} g|}$ factor multiplying $\mathcal{V}$, so the second term of (4.20) will also be unaffected by the Weyl rescaling if and only if $\mathcal{V}$ is a homogeneous function of degree one in the variables $Q_{1}$ and $\sqrt{Q_{2}}$.

We conclude that the weak-field and strong-field limits yield conformal theories of chiral 2-form electrodynamics. It is also true that these limits exhaust the possibilities

\footnotetext{
${ }^{11}$ Here we use the metric-independent alternating tensor density of weight -1 defined, in any coordinate system, by $\varepsilon_{012345}=1$.
} 
for conformal chiral 2-form electrodynamics. The reason is that conformal invariance requires $\mathcal{V}$ to be a homogeneous function of first degree in both $Q_{1}$ and $\sqrt{Q_{2}}$, in addition to satisfying (3.11), but this is equivalent to requiring the Hamiltonian density $\mathcal{H}(s, p)$ to be a homogeneous function of first degree in both arguments, in addition to satisfying (2.72). As a mathematical problem this is identical to the one already solved in [3] for $4 \mathrm{D}$ duality-invariant electrodynamics; the result in that case was that BB-electrodynamics and ModMax electrodynamics (including Maxwell) are the only possibilities.

A corollary of this result is that the pairing of $4 \mathrm{D}$ with $6 \mathrm{D}$ theories, of the type under discussion, is such that if one is conformal invariant then so is the other, and that all conformal electrodynamics theories (duality invariant in 4D and chiral in 6D) occur in $4 \mathrm{D} / 6 \mathrm{D}$ pairs.

\section{Higher dimensions: duality in $D=4 n$}

The study of non-linear $(2 n-1)$-form electrodynamics in a Minkowski spacetime of dimension $D=4 n$ for $n>1$, in particular the implications of an $\mathrm{SO}(2)$ or $\operatorname{Sl}(2 ; \mathbb{R})$ duality invariance, was initiated by Gibbons and Rasheed [7], who proposed an $n>1$ analog of the Born-Infeld Lagrangian, whose Hamiltonian form and strong-field limit were later found by Chruscinski [10].

As explained for $4 \mathrm{D}$ in section 3 , it is possible to make both Lorentz invariance and duality invariances manifest via a PST-type action that involves an additional non-null closed 1-form $v$ and a PST gauge invariance. Assuming that $v$ is timelike, as we shall do now, the Hamiltonian formulation is recovered on imposing the PST gauge $v=d t$. A higher-dimensional generalisation of this action was used in [8] to investigate possibilities for self-interactions for $n>1$ within the context of a perturbative expansion about the linear theory; in particular, new duality-invariant quartic self-interactions were found.

In this framework one starts from an $\mathrm{SO}(2)$ doublet of $(2 n-1)$-form gauge potentials $\left\{A^{\mathrm{a}} ; \mathrm{a}=1,2\right\}$ with $2 n$-form field strengths $F^{\mathrm{a}}=d A^{\mathrm{a}}$, which are used to define the following "electric" and "magnetic" $(2 n-1)$-form fields

$$
E^{\mathrm{a}}=i_{\hat{v}} F^{\mathrm{a}}, \quad B^{\mathrm{a}}=i_{\hat{v}} \widetilde{F}^{\mathrm{a}},
$$

where $\hat{v}$ is here the vector-field dual to the normalized 1 -form of (3.14); i.e. $\hat{v}^{2}=1$, and $\widetilde{F}^{\text {a }}$ is the Hodge dual of $F^{\mathrm{a}}$. The action for these fields, generalising (3.15), is

$$
I\left[A^{1}, A^{2}\right]=\int d^{4 n} x\left\{\frac{1}{2} \epsilon_{\mathrm{ab}} E^{\mathrm{a}} \cdot B^{\mathrm{b}}-\mathcal{H}\left(B^{1}, B^{2}\right)\right\},
$$

where

$$
E^{\mathrm{a}} \cdot B^{\mathrm{b}}=\frac{1}{(2 n-1) !}\left(E_{\mu_{1} \cdots \mu_{2 n-1}}\right)^{\mathrm{a}}\left(B^{\mu_{1} \cdots \mu_{2 n-1}}\right)^{\mathrm{b}},
$$

and $\mathcal{H}$ is a function of a basis for the independent $\mathrm{SO}(2)$-invariant Lorentz scalars formed with the $(2 n-1)$-forms $\left\{B^{1}, B^{2}\right\}$.

We shall find it convenient to use a notation in which the $k$ Lorentz indices of a $k$ form are denoted collectively by $[k]$. For example, in this notation the scalar product (5.3) 
becomes

$$
E^{\mathrm{a}} \cdot B^{\mathrm{b}}=\frac{1}{(2 n-1) !} E_{[2 n-1]}^{\mathrm{a}} B^{\mathrm{b}[2 n-1]} .
$$

Also, the partial derivatives of $\mathcal{H}$ are defined by

$$
d H=\frac{\partial \mathcal{H}}{\partial B^{\mathrm{a}}} \cdot d B^{\mathrm{a}}=\frac{1}{(2 n-1) !}\left(\frac{\partial \mathcal{H}}{\partial B_{[2 n-1]}^{\mathrm{a}}}\right) d B_{[2 n-1]}^{\mathrm{a}} .
$$

Using this notation the variation of the Lagrangian density induced by variation of the $(2 n-1)$-form potentials $A^{\text {a }}$ is, omitting a total derivative,

$$
\delta \mathcal{L}=\frac{1}{[(2 n-1) !]^{2}} \delta A_{[2 n-1]^{\prime}}^{\mathrm{c}} \epsilon_{\mathrm{ca}} \varepsilon^{\mu \nu[2 n-1]^{\prime}[2 n-1]} \partial_{\mu}\left[\Xi_{[2 n-1]}^{\mathrm{a}} \hat{\nu}_{\nu}\right]
$$

where the repeated indices of $[2 n-1]$ and $[2 n-1]^{\prime}$ are summed over, and

$$
\Xi_{[2 n-1]}^{\mathrm{a}}=E_{[2 n-1]}^{\mathrm{a}}-\epsilon^{\mathrm{ab}} \frac{\partial \mathcal{H}}{\partial B^{\mathrm{b}[2 n-1]}} .
$$

The equation of motion is therefore

$$
d\left[\Xi^{\mathrm{a}} \wedge \hat{v}\right]=0 .
$$

We remark that because $i_{v} B_{2 n-1}^{\mathrm{a}}=0$ and $\mathcal{H}$ is a Lorentz invariant function of $B_{2 n-1}^{\mathrm{a}}$, we have

$$
i_{v} \Xi^{\mathrm{a}}=0
$$

Recalling that $d v=0$, it is evident from (5.6) that the action (5.2) is invariant under the infinitesimal transformation

$$
\delta_{\phi} A^{\mathrm{a}}=v \wedge \phi^{\mathrm{a}}
$$

for $(2 n-2)$-form parameters $\phi^{\mathrm{a}}$; this is a generalization of the gauge invariance of (2.48) with gauge transformation (3.17). It may be used to 'gauge away' the exact form arising in the first-integral of (5.8), which thereby becomes equivalent to the $2 n$-form equation $\Xi^{\mathrm{a}} \wedge \hat{v}=0$. Because of (5.9) there is a further equivalence to $\Xi^{\mathrm{a}}=0$. To summarize, the field equation for the action (5.2) can be trivially once-integrated, and this first integral is gauge equivalent to the equation ${ }^{12}$

$$
\Xi^{\mathrm{a}}=E^{\mathrm{a}}-\epsilon^{\mathrm{ab}} \frac{\partial \mathcal{H}}{\partial B^{\mathrm{b}}}=0 .
$$

Generically, this is an equation not only for the field-strength $2 n$-forms $F^{\mathrm{a}}$ but also for the PST scalar $a$ appearing through its derivative $v=d a$, but there are special cases for which the action is invariant under the following 'first' PST gauge transformation (3.18) (analogous to $(3.10))$ :

$$
\delta_{\varphi} A^{\mathrm{a}}=-\frac{\varphi}{\sqrt{(\partial a)^{2}}} \Xi^{\mathrm{a}}, \quad \delta_{\varphi} a=\varphi
$$

\footnotetext{
${ }^{12}$ This non-linear generalization of a self-duality condition on $F^{\mathrm{a}}$ is also a generalization to $n \geq 1$ of the $n=1$ constitutive relations in the form given in (2.51).
} 
The action has this PST gauge invariance provided $\mathcal{H}$ also satisfies $[8,64]$

$$
\epsilon^{\mathrm{ab}} \varepsilon^{\mu \nu[.][\cdot]^{\prime}}\left(\frac{\partial \mathcal{H}}{\partial B^{\mathrm{a}[\cdot]}} \frac{\partial \mathcal{H}}{\partial B^{\mathrm{b}[\cdot]^{\prime}}}-B_{[\cdot]}^{\mathrm{a}} B_{[\cdot]^{\prime}}^{\mathrm{b}}\right)=0 .
$$

When this condition is satisfied the PST scalar field $a$ is a gauge degree of freedom that has no effect on the dynamics. In these cases the field equation for $a$ is

$$
\epsilon^{\mathrm{ab}} \varepsilon^{\mu \nu[2 n-1][2 n-1]^{\prime}} \partial_{\mu}\left(\frac{\partial_{\nu} a}{(\partial a)^{2}} \Xi_{[2 n-1]}^{\mathrm{a}} \Xi_{[2 n-1]^{\prime}}^{\mathrm{b}}\right)=0,
$$

which is identically satisfied when the gauge field equation (5.8) holds; this is the Noether identity guaranteed by Noether's second theorem.

\subsection{Generalized BI-type electrodynamics}

The condition (5.13) simplifies if one restricts $\mathcal{H}$ to be a function of the two Lorentz and $\mathrm{SO}(2)$-duality invariants

$$
s=-\frac{1}{2} B^{\mathrm{a}} \cdot B^{\mathrm{a}}, \quad q=\frac{1}{2} \epsilon_{\mathrm{ac}} \epsilon_{\mathrm{bd}}\left(B^{\mathrm{a}} \cdot B^{\mathrm{b}}\right)\left(B^{\mathrm{c}} \cdot B^{\mathrm{d}}\right),
$$

which generalize the $n=1$ duality invariant scalars of (3.19); in particular $q$ is invariant under the larger $S l(2 ; \mathbb{R})$ duality group. In this case, $(5.13)$ is equivalent to

$$
\mathcal{H}_{s}^{2}+4 s \mathcal{H}_{s} \mathcal{H}_{q}+4 q \mathcal{H}_{q}^{2}=1
$$

which is identical to (3.22); equivalently, to $(2.15)$ with $p=\sqrt{q}$.

Thus any solution of (5.16) yields a $(2 n-1)$-form electrodynamics not only for $n=1$ in $D=4$ but for any $n \geq 1$ in $D=4 n$. In particular, the solution

$$
\mathcal{H}=\sqrt{T^{2}+2 T\left((\cosh \gamma) s-(\sinh \gamma) \sqrt{s^{2}-q}\right)+q}-T
$$

for real parameter $\gamma$ yields a $D=4 n$ generalization of the generalized BI theory (4.8), which reduces for $\gamma=0$ to the Gibbons-Rasheed generalization of the BI theory [7]. The weak-field $(T \rightarrow \infty)$ limit, for any $\gamma$, is a generalization of the ModMax electrodynamics, see the Hamiltonian (4.9), which reduces to the free theory with $\mathcal{H}=s$ for $\gamma=0$. The strong-field limit is, independent of the value of $\gamma$,

$$
\mathcal{H}=\sqrt{q} .
$$

In the PST gauge $v=d t$, this becomes the $S l(2 ; \mathbb{R})$-invariant Hamiltonian density of Chruscinski's $D=4 n$ generalization of BB electrodynamics [10].

In a Lagrangian formulation of a duality invariant theory we need only a single $(2 n-1)$ form potential $A$ which will be identified with $A^{2}$. Thus $F:=d A \equiv d A^{2}$, and hence

$$
(E, B)=\left(E^{2}, B^{2}\right) .
$$

Notice that this choice accords, in the gauge $v=d t$, with the $n=1$ choice of (2.44). The Lagrangian density $\mathcal{L}$ must be a Lorentz scalar function of $(E, B)$, which is restricted by 
$\mathrm{SO}(2)$-duality invariance to satisfy a particular nonlinear differential equation [7]. This equation simplifies significantly if we restrict $\mathcal{L}$ to be a function of the Lorentz invariants

$$
\alpha=-\frac{1}{2}(E \cdot E-B \cdot B), \quad \beta=-(E \cdot B)^{2} .
$$

For $n=1$ (i.e. $D=4$ ) we have

$$
\alpha=S, \quad \beta=-P^{2},
$$

where $(S, P)$ are the Lorentz scalars of $(2.19)$, which provide a complete basis for any Lorentz scalar function; for $n>1$, functions of $(\alpha, \beta)$ constitute a special class of Lorentz scalar functions. Given that $\mathcal{L}=\mathcal{L}(\alpha, \beta)$, the condition for $\mathrm{SO}(2)$-duality invariance of the EL equations is

$$
\mathcal{L}_{\alpha}^{2}+4 \alpha \mathcal{L}_{\alpha} \mathcal{L}_{\beta}+4 \beta \mathcal{L}_{\beta}^{2}=1
$$

which is formally identical to (5.16). A family of solutions is

$$
\mathcal{L}(\alpha, \beta)=T-\sqrt{T^{2}-2 T\left[(\cosh \gamma) \alpha+(\sinh \gamma) \sqrt{\alpha^{2}-\beta}\right]+\beta} .
$$

For the case of $n=1$ and $\gamma=0$, it is not difficult to show that in the PST gauge $v=d t$, the function $\mathcal{H}$ of (5.17) is the BI Hamiltonian density, and the function $\mathcal{L}$ of (5.23) is the BI Lagrangian density, which is related to the Hamiltonian density by a Legendre transform with respect to $\mathbf{D}$; recall that $B^{1}$ is the 4 -vector with components $(0, \mathbf{D})$ for $n=1$ when $v=d t$.

More generally, we can define for all $n \geq 1$,

$$
\mathcal{L}(E, B)=\sup _{D}[-E \cdot D-\mathcal{H}(D, B)]
$$

where $D=B^{1}$. We shall assume that $\mathcal{H}(D, B)$ is a strictly convex function of $D$, so that $D$ is uniquely defined as a function of $E$ by

$$
E=-\frac{\partial \mathcal{H}(D, B)}{\partial D}
$$

In this case $\mathcal{L}$ will be a strictly convex function of $E$, which is a sufficient condition for the Legendre transform to be an involution. This guarantees equivalence of the Hamiltonian and Lagrangian field equations, and hence that the latter will be duality invariant if the former are duality invariant. However, it is impractical to find an explicit solution of (5.25) for $D$ as a function of $(E, B)$ when $\gamma \neq 0$.

The Legendre transform was found for the $n=1$ case in the weak-field limit (i.e. for the ModMax theory) by indirect means [3]; as expected, it is the weak-field limit of the generalized BI Lagrangian density of (4.13). We shall now show how this result can be extended beyond the weak-field limit to the full generalized BI theory for any $n \geq 1$.

\subsection{Legendre transform redux}

We first consider the general case for which $\mathcal{L}=\mathcal{L}(\alpha, \beta)$ and $\mathcal{H}=\mathcal{H}(s, q)$, assuming strict convexity; the results obtained will allow us to relate the Lagrangian and Hamiltonian 
densities of the generalized BI-type theories just discussed in the PST formulation. In the PST gauge $v=d t$, these functions become the conventional Lagrangian and Hamiltonian densities, so we are essentially implementing a Legendre transform but in a way that preserves manifest Lorentz invariance (although not manifest duality invariance).

Our starting point is the conjugate relation to (5.25):

$$
D=-\frac{\partial \mathcal{L}}{\partial E}=\mathcal{L}_{\alpha} E+2 B(E \cdot B) \mathcal{L}_{\beta} .
$$

Inserting this relation in (5.15) and (5.24) leads to [8]

$$
\begin{aligned}
s & =-\left(1+\mathcal{L}_{\alpha}^{2}-4 \beta \mathcal{L}_{\beta}^{2}\right) \kappa+\alpha \mathcal{L}_{\alpha}^{2}+2 \beta \mathcal{L}_{\alpha} \mathcal{L}_{\beta} \\
q & =\left(\beta-4 \alpha \kappa+4 \kappa^{2}\right) \mathcal{L}_{\alpha}^{2} \\
\mathcal{H} & =2(\alpha-\kappa) \mathcal{L}_{\alpha}+2 \beta \mathcal{L}_{\beta}-\mathcal{L}
\end{aligned}
$$

where

$$
\kappa=\frac{1}{2}(B \cdot B) .
$$

Now we take the differentials of these equations; i.e.

$$
d \mathcal{H}=2(\alpha-\kappa) d L_{\alpha}+2 \beta d L_{\beta}+L_{\alpha} d \alpha+L_{\beta} d \beta-2 L_{\alpha} d \kappa,
$$

and analogous expressions for $(d s, d q)$. By substitution for $d \mathcal{H}$ and $(d s, d q)$ one may verify that

$$
d \mathcal{H}=\left(\mathcal{L}_{\alpha}+4 \kappa \mathcal{L}_{\beta}\right)^{-1}\left[d s-\left(\frac{\mathcal{L}_{\beta}}{\mathcal{L}_{\alpha}}\right) d q\right] .
$$

The duality invariance condition (5.22) satisfied by $\mathcal{L}$ has ensured the absence of a $d \kappa$ term on the right hand side. From this result we deduce that

$$
\mathcal{H}_{s}=\left(\mathcal{L}_{\alpha}+4 \kappa \mathcal{L}_{\beta}\right)^{-1}, \quad \mathcal{H}_{q}=-\left(\frac{\mathcal{L}_{\beta}}{\mathcal{L}_{\alpha}}\right)\left(\mathcal{L}_{\alpha}+4 \kappa \mathcal{L}_{\beta}\right)^{-1}
$$

One may also verify that

$$
\sqrt{\alpha^{2}-\beta} \mathcal{L}_{\beta}=\sqrt{s^{2}-q} \mathcal{H}_{q}
$$

and that

$$
\mathcal{H}+\mathcal{L}=\sqrt{\left(s^{2}-q\right) \mathcal{H}_{s}^{2}+q}+\alpha \mathcal{L}_{\alpha}+2 \beta \mathcal{L}_{\beta} .
$$

The sign of the square roots in these equations may be checked by consideration of the free-field theory for which $\mathcal{H}=s$ and $\mathcal{L}=\alpha$. We note that both $\left(s^{2}-q\right)$ and $\left(\alpha^{2}-\beta\right)$ are non-negative.

Equations (5.33) implicitly determine $(\alpha, \beta)$ as functions of $(s, q)$ and, given these functions, equations (5.16) and (5.35) uniquely determine $\mathcal{H}$. Although we already know that the Legendre transform determines $\mathcal{H}$ implicitly, we now show how the new presentation of this fact allows us to obtain an explicit expression for the Hamiltonian density corresponding to the Lagrangian density of (5.23). For this case we find that

$$
\alpha \mathcal{L}_{\alpha}+2 \beta \mathcal{L}_{\beta}-\mathcal{L}=T\left[\cosh \gamma \sqrt{1+4\left(\alpha^{2}-\beta\right) \mathcal{L}_{\beta}^{2}}+2 \sinh \gamma \sqrt{\alpha^{2}-\beta} \mathcal{L}_{\beta}-1\right]
$$


where the duality-invariance condition on $\mathcal{L}$, in the form

$$
\left(\mathcal{L}_{\alpha}+2 \alpha \mathcal{L}_{\beta}\right)^{2}=1+4\left(\alpha^{2}-\beta\right) L_{\beta}^{2},
$$

has been used to obtain the first term in the bracket on the right hand side. Now, using both (5.34) and (5.35) we deduce that

$$
\mathcal{H}+T=\sqrt{\left(s^{2}-q\right) \mathcal{H}_{s}^{2}+q}+T\left[\cosh \gamma \sqrt{1+4\left(s^{2}-q\right) \mathcal{H}_{q}^{2}}+2 \sinh \gamma \sqrt{s^{2}-q} \mathcal{H}_{q}\right],
$$

which is a partial differential equation for $\mathcal{H}(s, q)$; taken together with (5.16), we have a system of two simultaneous differential equations with the unique solution

$$
\mathcal{H}=\sqrt{T^{2}+2 T\left[-(\sinh \gamma) \sqrt{s^{2}-q}+(\cosh \gamma) s\right]+q}-T .
$$

As expected, this is the Hamiltonian density of (5.17).

\section{Higher dimensions: chirality in $D=4 n+2$}

A chiral $2 n$-form electrodynamics is possible for $D=4 n+2$, and a manifestly Lorentz invariant action can be found by a straightforward generalization of the 6D PST action of (3.2). We introduce a $(2 n+1)$-form $H=d A$ and normalized PST 1-form $\hat{v}$, which we again assume to be timelike, and we again define 'electric' and 'magnetic' fields as

$$
\mathcal{E}=i_{\hat{v}} H, \quad \mathcal{B}=i_{\hat{v}} \tilde{H}
$$

where $\tilde{H}$ is the Hodge dual of $H$. The action is then

$$
S=\int d^{4 n+2} x\left(\frac{1}{2} \mathcal{E} \cdot \mathcal{B}-\mathcal{V}(\mathcal{B})\right)
$$

where we again use the notation

$$
\mathcal{E} \cdot \mathcal{B}=\frac{1}{(2 n) !} \mathcal{E}_{M_{1} \cdots M_{2 n}} \mathcal{B}^{M_{1} \cdots M_{2 n}},
$$

and similarly for any other pair of (2n)-forms; Lorentz indices have been raised here with a Minkowski metric of 'mostly-minus' signature. The PST gauge invariance of the action imposes the following condition on the 'potential' function $\mathcal{V}(\mathcal{B})[9]$ :

$$
\epsilon^{M N[\cdot][\cdot]^{\prime}}\left(\frac{\partial \mathcal{V}}{\partial \mathcal{B}[\cdot]} \frac{\partial \mathcal{V}}{\partial \mathcal{B}[\cdot]^{\prime}}-\mathcal{B}_{[\cdot]} \mathcal{B}_{[\cdot]^{\prime}}\right)=0
$$

where the notation is as in (5.5) and (5.13). In the $v=d t$ gauge $\mathcal{V}$ becomes the Hamiltonian density and this condition on it becomes the condition for Lorentz invariance of the phasespace action [11].

For $n=1$, the action (6.2) reduces to the 6D chiral 2-form action of (3.2) when account is taken of the fact that the 2 -form $B$ in that equation is $\sqrt{v^{2}} \mathcal{B}$. We have already discussed the possible choices for $\mathcal{V}$ in this $n=1$ case. For any $n>1$ there are two known possibilities: 
- Free-field theory [12]:

$$
\mathcal{V}=\frac{1}{2} \mathcal{B} \cdot \mathcal{B}
$$

This potential trivially satisfies (6.4).

- Strong-field theory [11]

$$
\mathcal{V}=\sqrt{-w^{2}}
$$

where $w$ is the $(4 n+2)$-vector

$$
w_{M}=-\frac{1}{2[(2 n) !]^{2}} \varepsilon_{M N M_{1} \cdots M_{2 n} N_{1} \cdots N_{2 n}} \mathcal{B}^{M_{1} \cdots M_{2 n}} \mathcal{B}^{N_{1} \cdots N_{2 n}} \hat{v}^{N} .
$$

We have verified that this potential is also a solution of (6.4).

For $n>1$ no other interacting theories are currently known, but some restrictions on the possibilities have been found [9].

The formula (6.7) implies that

$$
-w^{2}=\frac{(4 n) !}{4[(2 n) !]^{4}} \mathcal{B}^{P_{1} \cdots P_{2 n}} \mathcal{B}^{Q_{1} \cdots Q_{2 n}}\left[\mathcal{B}_{\left[P_{1} \cdots P_{2 n}\right.} \mathcal{B}_{\left.Q_{1} \cdots Q_{2 n}\right]}\right] .
$$

We give here relatively simple expressions for the $n=1,2$ cases:

$$
\begin{aligned}
& (n=1):-w^{2}=\frac{1}{2}(\mathcal{B} \cdot \mathcal{B})^{2}-\frac{1}{4} \mathcal{B}^{P Q} \mathcal{B}_{Q R} \mathcal{B}^{R S} \mathcal{B}_{S P}, \\
& (n=2):-w^{2}=\frac{1}{2}(\mathcal{B} \cdot \mathcal{B})^{2}-\frac{1}{2}\left(\mathcal{B}^{I} \mathcal{B}^{J}\right)\left(\mathcal{B}_{I} \mathcal{B}_{J}\right)+\frac{1}{16}\left(\mathcal{B}^{I J} \mathcal{B}^{K L}\right)\left(\mathcal{B}_{I J} \mathcal{B}_{K L}\right),
\end{aligned}
$$

where we have used the following notation for Lorentz tensors quadratic in $\mathcal{B}$ :

$$
\left(\mathcal{B}^{I} \mathcal{B}^{J}\right)=\frac{1}{3 !} \mathcal{B}^{I P Q R} \mathcal{B}^{J}{ }_{P Q R}, \quad\left(\mathcal{B}^{I J} \mathcal{B}^{K L}\right)=\frac{1}{2} \mathcal{B}^{I J P Q} \mathcal{B}^{K L}{ }_{P Q}
$$

\subsection{Reduction to $D=4 n$}

To perform the dimensional reduction/truncation to $D=4 n$ we split the $D=4 n+2$ Lorentz indices as follows

$$
M=(\mu, \mathrm{a}): \quad \mu=0,1, \cdots, 4 n ; \quad \mathrm{a}=1,2 .
$$

We then set to zero all components of $H$ except $H_{\mu_{1} \cdots \mu_{2 n} \text { a }}$, which we re-interpret as the components of a pair of $2 n$-forms $F_{\mathrm{a}}$, which we restrict to depend only on the coordinates of the $D=4 n$ Minkowski subspace. We also restrict the closed PST 1-form $v$ to this subspace, so $v \rightarrow d x^{\mu} v_{\mu}$. As a result

$$
\mathcal{E} \rightarrow E^{\mathrm{a}} \wedge d x_{\mathrm{a}}, \quad \mathcal{B} \rightarrow \epsilon_{\mathrm{ab}} \delta^{\mathrm{bc}} B^{\mathrm{a}} \wedge d x_{\mathrm{c}}, \quad \mathcal{V}(\mathcal{B}) \rightarrow \mathcal{H}\left(B^{1}, B^{2}\right) .
$$

The Euclidean metric on the 2-dimensional compact space appears in the reduction/truncation of $\mathcal{B}$ because this was defined using the Hodge dual. The fields $\left(E^{\mathrm{a}}, B^{\mathrm{b}}\right)$ on the $4 n$-dimensional Minkowski spacetime are independent of this 2-metric, so $\epsilon_{\mathrm{ab}} E^{\mathrm{a}} \cdot B^{\mathrm{b}}$ is an $S l(2 ; \mathbb{R})$ invariant. The action $(6.2)$ becomes the action (5.2), and the constraint (6.4) on $\mathcal{V}$ becomes the constraint $(5.13)$ on $\mathcal{H}$. In particular, $\mathcal{B} \cdot \mathcal{B} \rightarrow-B^{\mathrm{a}} \cdot B^{\mathrm{a}}$, which tells us that the truncation/reduction of the free chiral $2 n$-for electrodynamics in $D=4 n+2$ is the free duality invariant $(2 n-1)$-form electrodynamics in $D=4 n$. 


\subsubsection{A new $D>4$ generalization of $\mathrm{BB}$ electrodynamics}

The above reduction/truncation takes $d x^{M} w_{M} \rightarrow d x^{\mu} w_{\mu}$, where

$$
w_{\mu}=\frac{1}{2[(2 n-1) !]^{2}} \varepsilon_{\mathrm{ab}} \hat{v}^{\nu} \varepsilon_{\mu \nu \rho_{1} \cdots \rho_{2 n-1} \sigma_{1} \cdots \sigma_{2 n-1}}\left(B^{\rho_{1} \cdots \rho_{2 n-1}}\right)^{\mathrm{a}}\left(B^{\sigma_{1} \cdots \sigma_{2 n-1}}\right)^{\mathrm{b}}
$$

which yields

$$
-w^{2}=\frac{(4 n-2) !}{4[(2 n-1) !]^{4}} \epsilon^{\mathrm{ab}} \epsilon_{\mathrm{cd}} B_{\mathrm{a}}^{\mu_{1} \cdots \mu_{2 n-1}} B_{\mathrm{b}}^{\nu_{1} \cdots \nu_{2 n-1}}\left(B_{\left[\mu_{1} \cdots \mu_{2 n-1}\right.}^{\mathrm{c}} B_{\left.\nu_{1} \cdots \nu_{2 n-1}\right]}^{\mathrm{d}}\right)
$$

This Lorentz scalar is also manifestly $S l(2 ; \mathbb{R})$ invariant. We give here simplified expressions for $n=1,2$ :

- $n=1$.

$$
-w^{2}=B_{[\mathrm{a}}^{\mu} B_{\mathrm{b}]}^{\nu}\left(B_{\mu}^{\mathrm{a}} B_{\nu}^{\mathrm{b}}\right)=B^{2} D^{2}-(B \cdot D)^{2} .
$$

In the PST gauge $v=d t$ we have $\sqrt{-w^{2}}=|\mathbf{w}|=|\mathbf{D} \times \mathbf{B}|$, and hence BB electrodynamics.

- $n=2$

$$
-w^{2}=q+q^{\prime}
$$

where $q$ is the $S l(2 ; \mathbb{R})$ invariant of $(5.15)$, and

$$
q^{\prime}=-\frac{1}{8} \epsilon^{\mathrm{ab}} \epsilon_{\mathrm{cd}}\left(B_{\mathrm{a}}^{\mu \rho \sigma} B_{\nu \rho \sigma}^{\mathrm{c}}\right)\left(B_{\mathrm{b}}^{\nu \eta \tau} B_{\mu \eta \tau}^{\mathrm{d}}\right),
$$

which is another $S l(2 ; \mathbb{R})$ invariant.

From the result for $n=2$ we see that the reduction/truncation of the 'strong-field' chiral electrodynamics in $D=4 n+2$ yields an $S l(2 ; \mathbb{R})$-duality invariant electrodynamics in $D=8$ with

$$
\mathcal{H}=\sqrt{q+q^{\prime}} .
$$

This differs from the result (5.18) for the strong-field limit of the BI-type 8D dualityinvariant electrodynamics (and its one-parameter generalization) discussed in subsection 5.1. Moreover, the expression (6.15) shows that this difference will exist for all $n>1$ because the invariant $q$ is then just one term in the expansion of $(6.15)$ in $S l(2 ; \mathbb{R})$ invariants.

Ths inequivalence for $n>1$ of the strong-field limit [10] of the BI-type theories of [7] with the 'strong-field' theory obtained by reduction/truncation of the chiral 'strong-field' $D=4 n+2$ theory [11] is an indication that the strong-field limit of the BI-type theories can be 'lifted' to $D=4 n+2$ only for $n=1$. This would not be surprising because it is already known that the weak-field expansion of the BI-type theories is not the reduction/truncation of the weak-field expansion of any chiral $D=4 n+2$ theory [9]. 


\section{Summary and discussion}

We have explored multiple formulations of generic $\mathrm{SO}(2)$-duality invariant non-linear 4D electrodynamics theories and generic nonlinear 6D chiral 2-form electrodynamics. In each case, the generic model (whether $4 \mathrm{D}$ or $6 \mathrm{D}$ ) is determined by a function of two variables, and the condition for both Lorentz invariance and duality (4D) or chirality (6D) requires this function to satisfy a particular 'universal' PDE. Given a solution of this PDE, the PST method allows the construction of an action for which both Lorentz invariance and duality-invariance (4D) or chirality (6D) are manifest, but this depends on a new and nonmanifest (PST) gauge invariance; requiring this gauge invariance of the generic PST-type action again leads to the 'universal' PDE.

This $4 \mathrm{D} / 6 \mathrm{D}$ universality is partly explained by the fact that any chiral $6 \mathrm{D}$ 2-form electrodynamics theory contains, as a consistent reduction/truncation, a duality-invariant 4D electrodynamics theory. Here we have shown that this process also maps the $6 \mathrm{D}$ 'universal' $\mathrm{PDE}$ into the $4 \mathrm{D}$ 'universal' PDE. This implies a one-to-one correspondence between the sets of $4 \mathrm{D}$ and $6 \mathrm{D}$ theories since both are in one-to-one correspondence with the set of solutions to a single ' $4 \mathrm{D} / 6 \mathrm{D}$ universal' PDE: each solution yields both a duality invariant 4D electrodynamics and a $6 \mathrm{D}$ chiral 2-form electrodynamics related by reduction/truncation. The general solution of this PDE is known but various physical constraints (e.g. convexity, analyticity) mean that some solutions will have more physical relevance than others.

One well-studied solution yields both the $4 \mathrm{D}$ Born-Infeld theory, which arises in stringtheory as the effective dynamics on the worldvolume of a planar static D3-brane, and the chiral 2-form electrodynamics on the worldvolume of a planar static M5-brane. We have called this the "D3/M5 pair"; it is really a family of paired 4D/6D theories parameterized by a constant with dimensions of energy density. This family includes both a free-field limit, corresponding to weak fields with low energy density, and an interacting conformalinvariant strong-field limit. For 4D the strong-field limit is Bialynicki-Birula electrodynamics $[4,35]$, which is the unique $4 \mathrm{D}$ electrodynamics theory that is both Lorentz invariant and $S l(2 ; \mathbb{R})$-duality invariant. The corresponding $6 \mathrm{D}$ conformal chiral 2-form electrodynamics was found by Gibbons and West [5] as a limit (and truncation) of the M5 Hamiltonian density [13], and its 6D Lorentz invariance was established in [6].

One issue that we have clarified here is the relation between the Lagrangian and Hamiltonian formulations of Bialynicki-Birula electrodynamics. As shown in [4], the Lagrangian density found by Legendre transform of the Hamiltonian density is identically zero. Nevertheless, restrictions on the domain of this zero function contain the information that is required for reconstruction of the non-zero Hamiltonian density by a Legendre transform. A corollary of this result is that an alternative 'extended' Lagrangian, without restrictions on its domain, is a sum of constraints imposed by Lagrange multipliers, as originally proposed in [35]. Many readers will be familiar with the possibility that a non-trivial dynamical system may have a zero canonical Hamiltonian; Bialynicki-Birula electrodynamics shows how a non-trivial dynamical system may have a zero canonical Lagrangian.

Another solution of the 'universal' PDE yields, in 4D, the new one-parameter generalization of the Born-Infeld theory discussed in [3]; one of its features is that the weak-field 
and strong-field limits exhaust the possibilities for conformal invariant $4 \mathrm{D}$ electrodynamics. The strong field limit is the same as that of the Born-Infeld theory; i.e. Bialynicki-Birula electrodynamics. Its weak-field limit is a new one-parameter interacting generalization of Maxwell electrodynamics, which we called "ModMax" electrodynamics. This theory does not have an analytic Lagrangian density but the Hamiltonian density is analytic within its 'convexity domain' [3]. Here we have exhibited the corresponding family of $6 \mathrm{D}$ chiral 2 -form electrodynamics; in this case the weak-field limit is a conformal chiral 6D analog of the 4D ModMax theory, which it contains as a consistent reduction/truncation. Together, these $4 \mathrm{D} / 6 \mathrm{D}$ theories and their conformal limits constitute a 'generalized' D3/M5 pair.

There is a natural extension of $4 \mathrm{D}$ duality invariant nonlinear electrodynamics to duality-invariant $(2 n-1)$-form nonlinear electrodynamics in a Minkowski spacetime of dimension $D=4 n$, and a generalization of Born-Infeld theory to these dimensions has been proposed by Gibbons and Rasheed [7]; its strong-field limit is a generalization of BialynickiBirula electrodynamics that also has an enhanced $S l(2 ; \mathbb{R})$-duality invariance. It would be natural to suppose that this proposed generalization of Born-Infeld electrodynamics is the reduction/truncation of a chiral $2 n$-form electrodynamics in $D=4 n+2$ for $n>1$. For $n=2$, at least, we know that this supposition is false; this is because there is a unique quartic interaction in $10 \mathrm{D}$ and its reduction/truncation to $8 \mathrm{D}[9]$ has a different form to that appearing in a weak-field expansion of the Gibbons-Rasheed theory [7].

The only currently known 10D interacting chiral 4-form electrodynamics is the $n=2$ case of a class of conformal invariant chiral $2 n$-form electrodynamics in $D=4 n+2$ [11]. The $n=1$ case is the strong-field limit of the 'M5' chiral 2-form electrodynamics, so the $n=2$ case is a natural candidate for the strong-field limit of any proposed 10D generalization. Here we have shown that its truncation/reduction yields, as expected, an $8 \mathrm{D}$ conformal 3 -form electrodynamics with an enhanced $S l(2 ; \mathbb{R})$-duality invariance, but this is not the strong-field limit of the Gibbons-Rasheed theory. A corollary of these results is that, for $n>1$, not all $(2 n-1)$-form duality-invariant electrodynamics theories in $D=4 n$ are obtainable by reduction/truncation from a chiral $2 n$-form electrodynamics in $D=4 n+2$. In other words, chirality in $D=4 n+2$ implies duality in $D=4 n$ for all $n \geq 1$ but the one-toone correspondence between duality-invariant electrodynamics and chiral electrodynamics in space-time with two more spatial dimensions is a special feature of the $n=1$ case; in fact, the only known higher-dimensional $(n>1)$ analog of the interacting $4 \mathrm{D} / 6 \mathrm{D}$ pairs investigated in detail here is the one 'strong-field' case found here by reduction/truncation of the one known conformal 'strong-field' chiral electrodynamics in $D=4 n+2$. It is possible that this really is a strong-field limit of a new non-conformal $n>1$ pair but this remains to be determined.

Note added in proof. After this article was accepted for publication we became aware of $[81,82]$ where properties of a generic conformal (but not necessarily duality-invariant) non-linear $4 \mathrm{D}$ electrodynamics are studied. Birefringence properties were found in [81] with results for conformal theories in accord with those of [3]. The coupling to gravity was considered in [82], and it was shown that a certain condition on black hole charges restricts the form of the conformal electrodynamics Lagrangian density to a one-parameter 
extension of the Maxwell case. When expressed in terms of the Lorentz invariants $S$ and $P$ of our equation (2.20), this restricted Lagrangian density is

$$
\mathcal{L}(c)=\left(1-c^{2}\right) S+c^{2} \sqrt{S^{2}+P^{2}} \quad\left(c^{2} \leq 1\right) .
$$

For $2 c^{2} \leq 1$ we may write

$$
c^{2}=\frac{1}{2}\left(1-e^{-2 \gamma}\right) \quad(\gamma \geq 0)
$$

in which case

$$
\mathcal{L}(c)=e^{-\gamma} \mathcal{L}_{\operatorname{ModMax}}(\gamma)
$$

The constant factor of $e^{-\gamma}$ can be removed by a rescaling of the fields, so the restricted conformal electrodynamics Lagrangian density of [82] is, for $2 c^{2} \leq 1$ and prior to coupling to gravity, equivalent to the duality-invariant ModMax electrodynamics of [3], despite the fact that duality invariance was not assumed in [82].

\section{Acknowledgments}

IB and DS have been partially supported by the Spanish MICINN/FEDER (ERDF EU) grant PGC2018-095205-B-I00. The work of IB was also partially supported by the Basque Government Grant IT-979-16 and by the Basque Country University program UFI 11/55. PKT has been partially supported by STFC consolidated grant ST/L000385/1.

Open Access. This article is distributed under the terms of the Creative Commons Attribution License (CC-BY 4.0), which permits any use, distribution and reproduction in any medium, provided the original author(s) and source are credited.

\section{References}

[1] D. Berman, M5 on a torus and the three-brane, Nucl. Phys. B 533 (1998) 317 [hep-th/9804115] [INSPIRE].

[2] A. Nurmagambetov, Duality symmetric three-brane and its coupling to type IIB supergravity, Phys. Lett. B 436 (1998) 289 [hep-th/9804157] [INSPIRE].

[3] I. Bandos, K. Lechner, D. Sorokin and P.K. Townsend, A non-linear duality-invariant conformal extension of Maxwell's equations, Phys. Rev. D 102 (2020) 121703 [arXiv:2007.09092] [INSPIRE].

[4] I. Bialynicki-Birula, Nonlinear electrodynamics: variations on a theme by Born and Infeld, in Quantum theory of particles and fields: birthday volume dedicated to Jan Lopuszanski, B. Jancewicz and J. Lukierski eds., World Scientific, Singapore (1984), pg. 31.

[5] G.W. Gibbons and P.C. West, The metric and strong coupling limit of the M5-brane, J. Math. Phys. 42 (2001) 3188 [hep-th/0011149] [InSPIRE].

[6] P.K. Townsend, An interacting conformal chiral 2-form electrodynamics in six dimensions, Proc. Roy. Soc. Lond. A 476 (2020) 20190863 [arXiv:1911.01161] [InSPIRE]. 
[7] G.W. Gibbons and D.A. Rasheed, Electric-magnetic duality rotations in nonlinear electrodynamics, Nucl. Phys. B 454 (1995) 185 [hep-th/9506035] [INSPIRE].

[8] G. Buratti, K. Lechner and L. Melotti, Duality invariant self-interactions of Abelian p-forms in arbitrary dimensions, JHEP 09 (2019) 022 [arXiv: 1906.07094] [INSPIRE].

[9] G. Buratti, K. Lechner and L. Melotti, Self-interacting chiral p-forms in higher dimensions, Phys. Lett. B 798 (2019) 135018 [arXiv:1909.10404] [INSPIRE].

[10] D. Chruscinski, Strong field limit of the Born-Infeld p-form electrodynamics, Phys. Rev. D 62 (2000) 105007 [hep-th/0005215] [INSPIRE].

[11] P.K. Townsend, Manifestly Lorentz invariant chiral boson action, Phys. Rev. Lett. 124 (2020) 101604 [arXiv:1912.04773] [INSPIRE].

[12] M. Henneaux and C. Teitelboim, Dynamics of chiral (selfdual) p-forms, Phys. Lett. B 206 (1988) 650 [INSPIRE].

[13] E. Bergshoeff, D.P. Sorokin and P.K. Townsend, The M5-brane Hamiltonian, Nucl. Phys. B 533 (1998) 303 [hep-th/9805065] [INSPIRE].

[14] N. Marcus and J.H. Schwarz, Field theories that have no manifestly Lorentz invariant formulation, Phys. Lett. B 115 (1982) 111 [INSPIRE].

[15] M. Perry and J.H. Schwarz, Interacting chiral gauge fields in six-dimensions and Born-Infeld theory, Nucl. Phys. B 489 (1997) 47 [hep-th/9611065] [INSPIRE].

[16] P. Pasti, D.P. Sorokin and M. Tonin, Duality symmetric actions with manifest space-time symmetries, Phys. Rev. D 52 (1995) 4277 [hep-th/9506109] [INSPIRE].

[17] P. Pasti, D.P. Sorokin and M. Tonin, On Lorentz invariant actions for chiral p-forms, Phys. Rev. D 55 (1997) 6292 [hep-th/9611100] [InSPIRE].

[18] A. Maznytsia, C.R. Preitschopf and D.P. Sorokin, Duality of selfdual actions, Nucl. Phys. B 539 (1999) 438 [hep-th/9805110] [INSPIRE].

[19] M.K. Gaillard and B. Zumino, Duality rotations for interacting fields, Nucl. Phys. B 193 (1981) 221 [INSPIRE].

[20] S. Deser and O. Sarioglu, Hamiltonian electric/magnetic duality and Lorentz invariance, Phys. Lett. B 423 (1998) 369 [hep-th/9712067] [INSPIRE].

[21] X. Bekaert and M. Henneaux, Comments on chiral p-forms, Int. J. Theor. Phys. 38 (1999) 1161 [hep-th/9806062] [INSPIRE].

[22] X. Bekaert, Interactions of chiral two forms, PoS (tmr99) 007 (1999) [hep-th/9911109] [INSPIRE].

[23] R. Courant and D. Hilbert, Methods of mathematical physics: partial differential equations, volume 2, Interscience, (1962), pg. 91.

[24] M. Hatsuda, K. Kamimura and S. Sekiya, Electric magnetic duality invariant Lagrangians, Nucl. Phys. B 561 (1999) 341 [hep-th/9906103] [INSPIRE].

[25] R. Floreanini and R. Jackiw, Selfdual fields as charge density solitons, Phys. Rev. Lett. 59 (1987) 1873 [INSPIRE].

[26] M.K. Gaillard and B. Zumino, Selfduality in nonlinear electromagnetism, Lect. Notes Phys. 509 (1998) 121 [hep-th/9705226] [INSPIRE]. 
[27] M.K. Gaillard and B. Zumino, Nonlinear electromagnetic selfduality and Legendre transformations, in A Newton institute euroconference on duality and supersymmetric theories, (1997), pg. 33 [hep-th/9712103] [INSPIRE].

[28] S.M. Kuzenko and S. Theisen, Supersymmetric duality rotations, JHEP 03 (2000) 034 [hep-th/0001068] [INSPIRE].

[29] X. Bekaert and S. Cucu, Deformations of duality symmetric theories, Nucl. Phys. B 610 (2001) 433 [hep-th/0104048] [INSPIRE].

[30] E.A. Ivanov and B.M. Zupnik, New representation for Lagrangians of selfdual nonlinear electrodynamics, in $4^{\text {th }}$ international workshop on supersymmetry and quantum symmetries: $16^{\text {th }}$ Max Born symposium, (2002), pg. 235 [hep-th/0202203] [INSPIRE].

[31] E.A. Ivanov and B.M. Zupnik, New approach to nonlinear electrodynamics: dualities as symmetries of interaction, Phys. Atom. Nucl. 67 (2004) 2188 [Yad. Fiz. 67 (2004) 2212] [hep-th/0303192] [INSPIRE].

[32] S.M. Kuzenko, Manifestly duality-invariant interactions in diverse dimensions, Phys. Lett. B 798 (2019) 134995 [arXiv: 1908.04120] [INSPIRE].

[33] V. Arnold, Mathematical methods of classical mechanics, $2^{\text {nd }}$ edition, Springer, (1989).

[34] P.A.M. Dirac, Generalized Hamiltonian dynamics, Can. J. Math. 2 (1950) 129 [InSPIRE].

[35] I. Bialynicki-Birula, Field theory of photon dust, Acta Phys. Polon. B 23 (1992) 553 [INSPIRE].

[36] S. Deser and C. Teitelboim, Duality transformations of Abelian and non-Abelian gauge fields, Phys. Rev. D 13 (1976) 1592 [INSPIRE].

[37] L. Mezincescu and P.K. Townsend, DBI in the IR, J. Phys. A 53 (2020) 044002 [arXiv: 1907.06036] [INSPIRE].

[38] J.H. Schwarz and A. Sen, Duality symmetric actions, Nucl. Phys. B 411 (1994) 35 [hep-th/9304154] [INSPIRE].

[39] W. Siegel, Manifest Lorentz invariance sometimes requires nonlinearity, Nucl. Phys. B 238 (1984) 307 [INSPIRE].

[40] A.R. Kavalov and R.L. Mkrtchian, Lagrangian of the selfduality equation and $d=10, N=2 b$ supergravity, Sov. J. Nucl. Phys. 46 (1987) 728 [Yad. Fiz. 46 (1987) 1246] [inSPIRE].

[41] B. McClain, F. Yu and Y.S. Wu, Covariant quantization of chiral bosons and $\mathrm{OSp}(1,1 \mid 2)$ symmetry, Nucl. Phys. B 343 (1990) 689 [inSPIRE].

[42] C. Wotzasek, The Wess-Zumino term for chiral bosons, Phys. Rev. Lett. 66 (1991) 129 [INSPIRE].

[43] I. Bengtsson and A. Kleppe, On chiral p-forms, Int. J. Mod. Phys. A 12 (1997) 3397 [hep-th/9609102] [INSPIRE].

[44] N. Berkovits, Manifest electromagnetic duality in closed superstring field theory, Phys. Lett. B 388 (1996) 743 [hep-th/9607070] [INSPIRE].

[45] N. Berkovits, Local actions with electric and magnetic sources, Phys. Lett. B 395 (1997) 28 [hep-th/9610134] [INSPIRE].

[46] D. Belov and G.W. Moore, Holographic action for the self-dual field, hep-th/0605038 [INSPIRE]. 
[47] D.M. Belov and G.W. Moore, Type II actions from 11-dimensional Chern-Simons theories, hep-th/0611020 [INSPIRE].

[48] A. Sen, Covariant action for type IIB supergravity, JHEP 07 (2016) 017 [arXiv: 1511.08220] [INSPIRE].

[49] A. Sen, Self-dual forms: action, Hamiltonian and compactification, J. Phys. A 53 (2020) 084002 [arXiv: 1903.12196] [INSPIRE].

[50] K. Mkrtchyan, On covariant actions for chiral p-forms, JHEP 12 (2019) 076 [arXiv: 1908.01789] [INSPIRE].

[51] E. Andriolo, N. Lambert and C. Papageorgakis, Geometrical aspects of an Abelian $(2,0)$ action, JHEP 04 (2020) 200 [arXiv: 2003.10567] [INSPIRE].

[52] P. Vanichchapongjaroen, Covariant M5-brane action with self-dual 3-form, arXiv:2011.14384 [INSPIRE].

[53] A. Maznytsia, C.R. Preitschopf and D.P. Sorokin, Dual actions for chiral bosons, in $10^{\text {th }}$ summer school/seminar (Volga-10) on recent problems in theoretical and mathematical physics, (1998) [hep-th/9808049] [INSPIRE].

[54] S.-L. Ko and P. Vanichchapongjaroen, A covariantisation of M5-brane action in dual formulation, JHEP 01 (2018) 072 [arXiv: 1712.06408] [INSPIRE].

[55] D. Zwanziger, Local Lagrangian quantum field theory of electric and magnetic charges, Phys. Rev. D 3 (1971) 880 [INSPIRE].

[56] I.A. Bandos, K. Lechner, A. Nurmagambetov, P. Pasti, D.P. Sorokin and M. Tonin, Covariant action for the superfive-brane of M-theory, Phys. Rev. Lett. 78 (1997) 4332 [hep-th/9701149] [INSPIRE].

[57] M. Aganagic, J. Park, C. Popescu and J.H. Schwarz, World volume action of the M-theory five-brane, Nucl. Phys. B 496 (1997) 191 [hep-th/9701166] [INSPIRE].

[58] I. Bandos, On Lagrangian approach to self-dual gauge fields in spacetime of nontrivial topology, JHEP 08 (2014) 048 [arXiv: 1406.5185] [INSPIRE].

[59] H. Isono, Note on the self-duality of gauge fields in topologically nontrivial spacetime, PTEP 2014 (2014) 093B05 [arXiv: 1406.6023] [INSPIRE].

[60] P.S. Howe and E. Sezgin, $D=11, p=5$, Phys. Lett. B 394 (1997) 62 [hep-th/9611008] [INSPIRE].

[61] P.S. Howe, E. Sezgin and P.C. West, Covariant field equations of the M-theory five-brane, Phys. Lett. B 399 (1997) 49 [hep-th/9702008] [InSPIRE].

[62] P.S. Howe, E. Sezgin and P.C. West, The six-dimensional selfdual tensor, Phys. Lett. B 400 (1997) 255 [hep-th/9702111] [INSPIRE].

[63] I.A. Bandos, K. Lechner, A. Nurmagambetov, P. Pasti, D.P. Sorokin and M. Tonin, On the equivalence of different formulations of the M-theory five-brane, Phys. Lett. B 408 (1997) 135 [hep-th/9703127] [INSPIRE].

[64] P. Pasti, D. Sorokin and M. Tonin, Covariant actions for models with non-linear twisted self-duality, Phys. Rev. D 86 (2012) 045013 [arXiv: 1205.4243] [INSPIRE].

[65] C. Lee and H. Min, SL $(2, R)$ duality-symmetric action for electromagnetic theory with electric and magnetic sources, Annals Phys. 339 (2013) 328 [arXiv:1306.5520] [INSPIRE]. 
[66] P. Vanichchapongjaroen, Dual formulation of covariant nonlinear duality-symmetric action of kappa-symmetric D3-brane, JHEP 02 (2018) 116 [arXiv:1712.06425] [INSPIRE].

[67] S.-L. Ko and P. Vanichchapongjaroen, The dual formulation of M5-brane action, JHEP 06 (2016) 022 [arXiv: 1605.04705] [inSPIRE].

[68] S.-L. Ko, D. Sorokin and P. Vanichchapongjaroen, The M5-brane action revisited, JHEP 11 (2013) 072 [arXiv: 1308.2231] [INSPIRE].

[69] P.-M. Ho and Y. Matsuo, M5 from M2, JHEP 06 (2008) 105 [arXiv:0804.3629] [INSPIRE].

[70] P.-M. Ho, Y. Imamura, Y. Matsuo and S. Shiba, M5-brane in three-form flux and multiple M2-branes, JHEP 08 (2008) 014 [arXiv: 0805.2898] [INSPIRE].

[71] I.A. Bandos and P.K. Townsend, Light-cone M5 and multiple M2-branes, Class. Quant. Grav. 25 (2008) 245003 [arXiv:0806 . 4777] [INSPIRE].

[72] I.A. Bandos and P.K. Townsend, SDiff gauge theory and the M2 condensate, JHEP 02 (2009) 013 [arXiv: 0808.1583] [InSPIRE].

[73] P. Pasti, I. Samsonov, D. Sorokin and M. Tonin, BLG-motivated Lagrangian formulation for the chiral two-form gauge field in $D=6$ and M5-branes, Phys. Rev. D 80 (2009) 086008 [arXiv: 0907.4596] [INSPIRE].

[74] B.P. Kosyakov, Nonlinear electrodynamics with the maximum allowable symmetries, Phys. Lett. B 810 (2020) 135840 [arXiv:2007.13878] [INSPIRE].

[75] D. Flores-Alfonso, B.A. González-Morales, R. Linares and M. Maceda, Black holes and gravitational waves sourced by non-linear duality rotation-invariant conformal electromagnetic matter, Phys. Lett. B 812 (2021) 136011 [arXiv:2011.10836] [INSPIRE].

[76] A.B. Bordo, D. Kubiznak and T.R. Perche, Taub-NUT solutions in conformal electrodynamics, arXiv:2011.13398 [INSPIRE].

[77] D. Flores-Alfonso, R. Linares and M. Maceda, Nonlinear extensions of gravitating dyons: from NUT wormholes to Taub-Bolt instantons, arXiv:2012.03416 [INSPIRE].

[78] Z. Amirabi and S.H. Mazharimousavi, Black-hole solution in nonlinear electrodynamics with the maximum allowable symmetries, arXiv:2012.07443 [INSPIRE].

[79] B. Zumino, Effective Lagrangians and broken symmetries, in Lectures on elementary particles and quantum field theory, volume 2, Brandeis Univ., Cambridge, MA, U.S.A. (1970), pg. 437.

[80] S. Faci, Conformal invariance: from Weyl to SO(2, d), EPL 101 (2013) 31002 [arXiv: 1206.4362] [INSPIRE].

[81] V.I. Denisov, E.E. Dolgaya, V.A. Sokolov and I.P. Denisova, Conformal invariant vacuum nonlinear electrodynamics, Phys. Rev. D 96 (2017) 036008 [inSPIRE].

[82] I.P. Denisova, B.D. Garmaev and V.A. Sokolov, Compact objects in conformal nonlinear electrodynamics, Eur. Phys. J. C $\mathbf{7 9}$ (2019) 531 [arXiv:1901.05318] [INSPIRE]. 